\title{
Resource Efficiency: A New Paradigm on Energy Efficiency and Spectral Efficiency Tradeoff
}

\author{
Jie Tang, Member, IEEE, Daniel K. C. So, Member, IEEE, Emad Alsusa, Senior Member, IEEE and \\ Khairi Ashour Hamdi, Senior Member, IEEE
}

\begin{abstract}
Spectral efficiency (SE) and energy efficiency (EE) are the main metrics for designing wireless networks. Rather than focusing on either SE or EE separately, recent works have focused on the relationship between $E E$ and $S E$ and provided good insights into the joint EE-SE tradeoff. However, such works have assumed that bandwidth are fully occupied regardless of the transmission requirements and therefore are only valid for this scenario. In this paper, we propose a new paradigm for EE-SE tradeoff, namely the resource efficiency (RE) for orthogonal frequency division multiple access (OFDMA) cellular network in which we take into consideration different transmission-bandwidth requirements. We analyse the properties of the proposed $R E$ and prove that it is capable of exploiting the tradeoff between EE and SE by balancing consumption power and occupied bandwidth; hence simultaneously optimizing both EE and SE. We then formulate the generalized RE optimization problem with guaranteed quality of service $(\mathrm{QOS})$ and provide a gradient based optimal power adaptation scheme to solve it. We also provide an upper bound near optimal method to jointly solve the optimization problem. Furthermore, a low-complexity suboptimal algorithm based on uniform power allocation scheme is proposed to reduce the complexity. Numerical results confirm the analytical findings and demonstrate the effectiveness of the proposed resource allocation schemes for efficient resource usage.
\end{abstract}

Index Terms-Green radio (GR), orthogonal frequency division multiple access (OFDMA), resource efficiency (RE), energy efficiency (EE), spectral efficiency (SE).

\section{INTRODUCTION}

One of the major challenges in wireless communications is the increased energy consumption as a result of the rising number of emerging high data rate wireless applications such as multimedia and interactive services. Excessive power usage in such networks is a critical issue for the mobile operators in particular. To meet these challenges, the green evolution has become an urgent need for wireless networks today. Green radio, a research direction for the evolution of future wireless architectures and techniques, has thereby been pursued as an important trend for future energy-efficient wireless communications.

Up to now, the spectral efficiency (SE) metric has been the main performance indicator for designing and optimizing wireless communication networks. It is an important measure for quantitatively evaluating the effectiveness of cellular systems and has been extensively studied for various scenarios

J. Tang, D. So, E. Alsusa and K. Hamdi are with the School of Electrical and Electronics Engineering, The University of Manchester, Manchester, M13 9PL, UK, Emails: \{jie.tang, d.so, e.alsusa, k.hamdi\}@manchester.ac.uk.

This work has been supported by the Engineering and Physical Sciences Research Council of UK, under grant EP/J021768/1.
[1]-[3]. Although SE measures how efficiently a limited frequency spectrum is utilized, it fails to account for how efficiently power is consumed. Due to high network power consumption, research on energy efficiency (EE) has attracted much interests recently, including single link optimization [4], single cell scenario [5], multi-cell deployment [6], cognitive radio network [7] and cooperative relaying network [8].

Orthogonal frequency division multiple access (OFDMA) has been recognized as one of the best candidates for next generation broadband wireless standards because of its natural ability to utilize different portions of the spectrum. In OFDMA networks, multiple users share the total bandwidth to exploit multiuser diversity and improve the system capacity with dynamic resource allocation strategies. OFDMA has been extensively studied from the SE perspective. Recently, more attention has been paid to energy-efficient design in OFDMA networks. Energy-efficient OFDMA is first addressed in [9] which shows at least a $20 \%$ reduction in power consumption when performing EE optimization. Authors in [10] studied resource allocation for energy-efficient communication in multicell orthogonal OFDMA downlink networks with cooperative base stations, and extended the work to the case of a large number of transmit antennas in [11]. Energy-efficient design for multi-user OFDMA has also been studied. Energy-efficient radio resource scheduling with quality of service (QoS) guarantees in a multi-user OFDMA system has been studied in [12]. An optimization problem is formulated to maximize energy efficiency subject to users QoS requirements, and addressed using Lagrangian duality. Authors in [13] developed a low complexity energy-efficient scheduling scheme for uplink OFDMA scenario. The problem of resource allocation for the uplink of a multicell wireless network has been studied in [14]. Furthermore, downlink scenario has also been investigated. Energy-efficient design for downlink OFDMA with delaysensitive traffic has been studied in [15]. In [16], an energyefficient resource allocation scheme in both downlink and uplink OFDMA networks has been studied.

Unfortunately, optimizing EE and SE do not always coincide and may even conflict sometimes [17], [18]. Hence, how to balance EE and SE is a problem well worth studying. Conventional design of wireless networks mainly focuses on either SE or EE. Recently, the relationship between EE and SE has become an important research topic. In [19], a framework to integrate the connections between $\mathrm{EE}$ and SE tradeoff has been proposed. Authors in [20] investigates the tradeoff between EE and SE for interference-limited wireless networks. In [21], EE-SE tradeoff with consideration of circuit power 
has been studied for energy-constrained wireless multihop networks with a single source-destination pair. In [22], a generic closed-form approximation of the EE-SE tradeoff has been derived for the uplink of coordinated multi-point (CoMP) system. Authors in [23] investigated the EE-SE relationship in a single cell downlink OFDMA network, which is important for designing energy efficient networks that require a better balance between EE and SE. They proved that the EE-SE relationship is a quasiconcave function. Based on that, a tight lower bound and a tight upper bound on the EE-SE curve are provided by Lagrange dual decomposition (LDD) and continuous relaxation, respectively. However, all such works about EE-SE tradeoff assumed that bandwidth are fully occupied regardless of the transmission requirements. As a result, this may lead to inefficient use of network resources.

\section{A. Main Contributions}

In this paper, we propose a new paradigm for EE-SE tradeoff, namely the resource efficiency (RE) for OFDMA cellular network which accounts for all scenarios of bandwidth loading. We analyze the properties of the proposed RE and prove that it can exploit the tradeoff between EE and SE. We then formulate the generalized RE optimization problem for the downlink transmission with guaranteed QoS as a multiobjective optimization problem. This optimization problem is in general NP-hard for the optimal solution. To obtain insight into the problem, we first investigate the properties of the case with a given subcarrier assignment and obtained an analytical solution. By using water-filling algorithm for power allocation, we developed a gradient based optimal power adaptation scheme to solve the resource allocation problem. Since the optimal solution needs to check all possible number of active subcarriers, and for each case apply the waterfilling algorithm and gradient updated scheme to find the optimal power allocation, the complexity is comparatively high however. We also provide an upper bound near optimal method to jointly solve the optimization problem based on interior point method. Although convex programming is numerically stable, its computational complexity depends on the number of optimizing variables, which can be large if the number of subcarriers and/or the number of UEs is still large. Therefore, we further explore the property of the optimization problem using the uniform power allocation scheme and propose a novel low-complexity suboptimal algorithm to solve the original problem. Simulation results confirm the theoretical findings and reveal that by allowing a slight increase in energy consumption (or bandwidth usage), the proposed approach can significantly reduce the amount of bandwidth used (or energy consumed). Thus the proposed RE can efficiently optimize the use of available resources in the network.

\section{B. Organization and Notation}

The rest of this paper is organized as follows. The system model and the limitation for energy-efficient or spectralefficient only design are described in Section II. In Section III, we introduce the proposed metric description and formulate the optimization problem. In Section IV, we first develop a gradient based optimal power adaptation scheme to solve the resource allocation problem. We then provide an upper bound near optimal method to jointly solve the optimization problem. To reduce complexity, a novel low-complexity suboptimal algorithm is also proposed to solve the original problem. Simulation results are provided in Section $\mathrm{V}$ and conclusions are drawn in Section VI.

The following notations are used in the paper. Bold upper and lower case letters denote matrices and vectors, respectively; $[x]^{+}$denotes $\max (x, 0)$ and $[\cdot]_{K \times N}$ denotes an $K \times N$ matrix.

\section{SYSTEM MODEL}

In this section, we first introduce the system model and then discuss the limitation for energy-efficient or spectral-efficient only design.

\section{A. System Description}

Consider a single cell downlink OFDMA network with $K$ active user equipments (UE). The total bandwidth, $W_{t o t}$, is divided into $N$ subcarriers, each with a bandwidth of $W_{C}=$ $\frac{W_{t o t}}{N}$. We assume that subcarriers are allocated centrally and each subcarrier cannot be assigned to more than one user to avoid interference among different UEs. The transmit power and the channel frequency response of the $k^{t h} \mathrm{UE}$ on the $n^{t h}$ subcarrier are denoted as $p_{k, n}$ and $H_{k, n}$, respectively. Assuming perfect channel state information in both transmitter and receiver, the maximum achievable data rate of the $k^{\text {th }} \mathrm{UE}$ on the $n^{\text {th }}$ subcarrier is accordingly

$$
r_{k, n}=W_{C} \log _{2}\left(1+\frac{p_{k, n}\left|H_{k, n}\right|^{2}}{N_{0} W_{C}}\right)
$$

where $N_{0}$ is the single-sided noise spectral density. Hence, the aggregate rate for the $k^{t h} \mathrm{UE}$ and the overall throughput are shown respectively as follows

$$
\begin{gathered}
R_{k}=\sum_{n \in \mathcal{N}} \rho_{k, n} r_{k, n} \\
R=\sum_{k \in \mathcal{K}} R_{k}=\sum_{k \in \mathcal{K}} \sum_{n \in \mathcal{N}} \rho_{k, n} r_{k, n}
\end{gathered}
$$

where $\rho_{k, n} \in\{1,0\}$ indicates whether or not the $n^{\text {th }}$ subcarrier is assigned to the $k^{t h} \mathrm{UE}, \mathcal{N}=\{1,2, \cdots, N\}$ and $\mathcal{K}=\{1,2, \cdots, K\}$ denote the sets of all subcarriers and all UEs, respectively. Obviously, a feasible subcarrier assignment indicator matrix, $\boldsymbol{\rho}=\left[\rho_{k, n}\right]_{K \times N}$, should satisfy

$$
\begin{gathered}
\sum_{k \in \mathcal{K}} \rho_{k, n} \leq 1, \forall n \in \mathcal{N} \\
\rho_{k, n} \in\{0,1\}, \forall k \in \mathcal{K}, \forall n \in \mathcal{N} \\
\sum_{k \in \mathcal{K}} \sum_{n \in \mathcal{N}} \rho_{k, n}=Q
\end{gathered}
$$

where $Q$ denotes the number of used subcarriers, and hence $Q \leq N$. $\mathcal{Q}$ is defined as the sets of used subcarriers, $\mathcal{Q} \subseteq$ $\mathcal{N}$. The subcarrier assignment constraint in (4)-(6) can be 


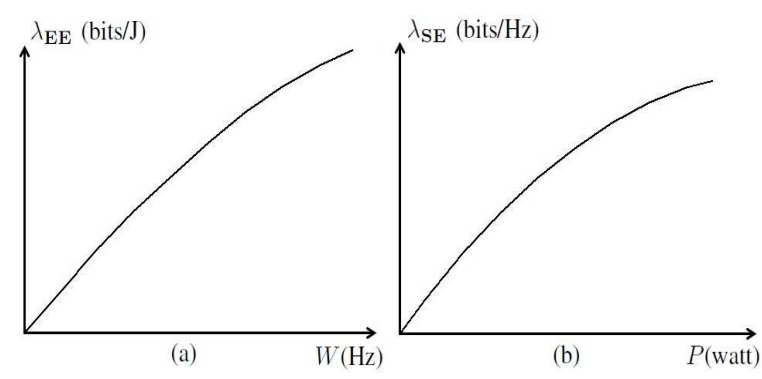

Fig. 1. $\quad$ EE and SE versus bandwidth $W$ and power $P$.

equivalently viewed from the following perspective

$$
\bigcup_{k=1}^{K} \mathcal{S}_{k} \subseteq \mathcal{Q} \text { and } \mathcal{S}_{k} \bigcap \mathcal{S}_{k^{\prime}}=\emptyset, \forall k \neq k^{\prime},
$$

where $\mathcal{S}_{k}$ is the set of subcarriers assigned to the $k^{t h}$ UE. Since the total transmit power of either base station or UE is nonnegative and also limited, any possible power allocation matrix, $\mathbf{P}_{\mathbf{T}}=\left[p_{k, n}\right]_{K \times N}$, should satisfy the following conditions

$$
\begin{gathered}
p_{k, n} \geq 0, \forall k \in \mathcal{K}, \forall n \in \mathcal{N} \\
\sum_{k \in \mathcal{K}} \sum_{n \in \mathcal{N}} p_{k, n} \leq P_{\max }
\end{gathered}
$$

where $P_{\max }$ represent the maximum total transmit power at the base station for downlink transmission.

The overall transmit power for the $k^{t h} \mathrm{UE}$ and the total transmit power are shown as follows

$$
\begin{gathered}
P_{k}=\sum_{n \in \mathcal{N}} p_{k, n} \\
P_{T}=\sum_{k \in \mathcal{K}} P_{k}=\sum_{k \in \mathcal{K}} \sum_{n \in \mathcal{N}} p_{k, n} .
\end{gathered}
$$

In addition to transmit power, other active circuit blocks also consume energy [24]. For the downlink transmission, the overall power consumption model at the base station is given by [24]

$$
P=\zeta P_{T}+P_{C}
$$

where $\zeta$ is the reciprocal of drain efficiency of power amplifier and $P_{C}$ represents the circuit power. Similarly, the overall power budget at the base station is modeled as

$$
P_{t o t}=\zeta P_{\max }+P_{C} .
$$

\section{B. Energy Efficiency}

Conventional EE for downlink transmission is defined as the total delivered bits per unit energy, where energy consumption includes transmission energy consumption and circuit energy consumption in active mode, i.e.,

$$
\lambda_{E E} \triangleq \frac{R}{P}=\frac{\sum_{k \in \mathcal{K}} \sum_{n \in \mathcal{N}} \rho_{k, n} r_{k, n}}{\zeta P_{T}+P_{C}} .
$$

Since EE represents the delivered bits per unit energy, optimizing EE has its limitation. As shown in Fig. 1(a), system will occupy as much bandwidth as possible to maximize the energy efficiency. Hence it reduces the efficient use of resource in terms of bandwidth usage. Therefore, EE has its limitation in terms of optimally using the existing resources.

\section{Spectral Efficiency}

On the other hand, SE is defined as the total (average) number of delivered bits per unit bandwidth.

$$
\lambda_{S E} \triangleq \frac{R}{W}=\frac{\sum_{k \in \mathcal{K}} \sum_{n \in \mathcal{N}} \rho_{k, n} r_{k, n}}{W}
$$

where $W$ represent the occupied bandwidth. In OFDMA network, the occupied bandwidth relates to the number of active subcarriers,

$$
W=Q \times W_{C}=Q \times \frac{W_{t o t}}{N} .
$$

Similar to EE, optimizing SE also has its limitation. It will use as much power as possible to maximize the spectral efficiency which is shown in Fig. 1(b), and hence reduces the resource utilization in terms of power usage.

\section{RESOURCE EFFICIENCY AND PROBLEM FORMULATION}

As the conventional system design using $\mathrm{EE}$ or SE has limitation in terms of inefficient use of resources, we therefore introduce multi-objective optimization to optimize both $\mathrm{EE}$ and SE. In general, a constrained multi-objective optimization problem is defined as follows [25]:

$$
\begin{array}{ll}
\min _{\mathbf{x}} & F(\mathbf{x})=\left(f_{1}(\mathbf{x}), f_{2}(\mathbf{x}), \cdots, f_{k}(\mathbf{x})\right) \\
\text { s.t. } & g_{i}(\mathbf{x}) \leq 0, i=1,2, \cdots, m, \\
& h_{j}(\mathbf{x})=0, j=1,2, \cdots, n,
\end{array}
$$

where $F(\mathbf{x})$ is the set of objective functions, $g_{i}(\mathbf{x})$ is the set of inequality constraints and $h_{j}(\mathbf{x})$ is the set of equality constraints. A constrained multi-objective optimization problem minimizes $k$ objective functions simultaneously, where the objective functions represent (usually) competing or conflicting objectives. A multi-objective problem is often solved by combining its multiple objectives into a single-objective scalar function. This approach is in general known as the weightedsum or scalarization method. In particular, the weighted-sum method minimizes a positively weighted convex sum of the objectives, that is

$$
\begin{array}{ll}
\min _{\gamma_{l}, \mathbf{x}} & \sum_{l=1}^{k} \gamma_{l} f_{l}(\mathbf{x}) \\
\text { s.t. } & \sum_{l=1}^{k} \gamma_{l}=1, \gamma_{l}>0, l=1,2, \cdots, k, \\
& g_{i}(\mathbf{x}) \leq 0, i=1,2, \cdots, m,
\end{array}
$$

that represents a new optimization problem with a unique objective function. It can be proved that the minimizer of this single-objective function is an efficient solution for the original multi-objective problem [25], i.e., its image belongs to the Pareto curve.

In this work, since both EE and SE are our research target, we consider both of them as our objective functions 
and transformed the constrained multi-objective optimization problem to single-objective function as follows,

$$
\begin{array}{ll}
\max & \gamma_{1} \lambda_{E E}+\gamma_{2} \lambda_{S E} \\
\text { s.t. } & \gamma_{1}+\gamma_{2}=1, \\
& \gamma_{1}>0, \gamma_{2}>0 .
\end{array}
$$

However, the unit for EE is bits/Joule while the unit for SE is bits $/ \mathrm{s} / \mathrm{Hz}$, and hence it is inappropriate to directly add $\mathrm{EE}$ and SE due to inconsistence of metric unit. Moreover, since the bandwidth $W$ is numerically larger then the transmission power $P_{T}$, a simple summation of $\mathrm{EE}$ and SE will tend to focus the optimization problem on EE. This will defeat the purpose of balancing between EE and SE. As a result, we now introduce a new system metric called resource efficiency defined as

$$
\lambda_{R E} \triangleq \frac{R}{P}\left(1+\beta \frac{\eta_{P}}{\eta_{W}}\right)
$$

where $\eta_{P}$ and $\eta_{W}$ represent power utilization and bandwidth utilization respectively, given by

$$
\eta_{P} \triangleq \frac{P}{P_{t o t}}, \quad \eta_{W} \triangleq \frac{W}{W_{t o t}} .
$$

These variables are to denote the ratio of power and bandwidth used out of those that are available. $\beta$ is a weighted factor to control the balance of EE and SE. In the following, we show that this new system metric is capable of exploiting the tradeoff between EE and SE by balancing consumption power and occupied bandwidth.

Property I. Resource efficiency is capable of exploiting the tradeoff between $E E$ and $S E$, with the weighting between them controlled by $\beta \frac{W_{t o t}}{P_{t o t}}$.

Proof: By substituting (27) into (26), we have

$$
\begin{aligned}
\lambda_{R E} & =\frac{R}{P}\left(1+\beta \frac{\frac{P}{P_{t o t}}}{\frac{W}{W_{t o t}}}\right) \\
& =\frac{R}{P}+\beta \frac{R}{P} \frac{P}{P_{t o t}} \frac{W_{t o t}}{W} \\
& =\frac{R}{P}+\beta \frac{R}{W} \frac{W_{t o t}}{P_{t o t}} \\
& =\lambda_{E E}+\bar{\beta} \lambda_{S E} .
\end{aligned}
$$

where $\bar{\beta}=\beta \frac{W_{t o t}}{P_{t o t}}$. Hence RE can be rewritten as a combination of EE and SE, $\frac{W_{t o t}}{P_{t o t}}$ acts as unit normalizer for SE and $\mathrm{EE}$ while $\beta$ acts as the weighted factor to control the balance of EE and SE. Furthermore, $\bar{\beta}$ in the proposed RE is equal to $\frac{\gamma_{2}}{\gamma_{1}}$ in the scalarization method in (23)-(25).

Interestingly, due to the normalization factor $\frac{W_{t o t}}{P_{t o t}}$, the unit of the proposed RE is the same as EE which is still bits/Joule, which is a good measure for green communications. Furthermore, considering a fixed of $\beta$, when the total bandwidth $W_{t o t}$ is larger than the total power budget $P_{t o t}$, RE emphasis more on SE. On the other hand when the total bandwidth $W_{\text {tot }}$ is smaller compared to the total power budget $P_{t o t}$, RE will put more weight on EE. Practically, it reveals that RE will emphasis more on SE to save occupied bandwidth when the system has sufficient bandwidth to support the transmission requirement. For this case, if $\mathrm{RE}$ emphasize more on $\mathrm{EE}$, the system will occupy as much bandwidth as possible to maximize the $\mathrm{EE}$ and hence reduces the efficient use of resource in terms of bandwidth usage. On the other hand, RE will put more weight on EE to save transmission power when the system has ample power to support the transmission requirement. For this case, if RE emphasize more on SE, the system will use as much power as possible to maximize SE and hence reduce the green resource usage in terms of power usage. Consequently, this proposed system metric is capable of exploiting the tradeoff between EE and SE and adapt its optimization based on the available resources.

On the other hand, $\beta$ is the weight between $\mathrm{EE}$ and SE such that RE optimize EE when $\beta=0$ but optimize SE when $\beta=\infty$. There is no a-priori correspondence between a weight vector and a solution vector and hence it is up to the decision maker to choose appropriate weights. Consequently, without loss of generality, we consider $\beta$ as a constant in our RE optimization problem. Since providing different service priorities and guaranteeing QoS for each UE is important in communication system design, we consider the generalized RE under a series of traffic-related minimum rate requirements, $\gamma_{k}$, and the peak transmit power, $P_{\max }$. Hence, the generalized RE optimization problem for the downlink transmission can be mathematically formulated as follows

$$
\max _{\boldsymbol{\rho}, \mathbf{P}_{\mathbf{T}}} \frac{\sum_{k \in \mathcal{K}} \sum_{n \in \mathcal{N}} \rho_{k, n} r_{k, n}}{\zeta P_{T}+P_{C}}\left(1+\beta \frac{\eta_{P}}{\eta_{W}}\right)
$$

subject to

$$
\begin{gathered}
\sum_{n \in \mathcal{N}} \rho_{k, n} r_{k, n} \geq \gamma_{k}, \forall k \in \mathcal{K} \\
\sum_{k \in \mathcal{K}} \sum_{n \in \mathcal{N}} p_{k, n} \leq P_{\text {max }} \\
\sum_{k \in \mathcal{K}} \sum_{n \in \mathcal{N}} \rho_{k, n} \leq N .
\end{gathered}
$$

\section{The Proposed optimal AND NEAR-OPTIMAL RESOURCE ALLOCATION SCHEMES}

In this section, we will develop a gradient based optimal power adaptation scheme and a near optimal upper bound approach based on interior point method for the resourceefficient resource allocation in the downlink transmission.

\section{A. Optimal resource allocation scheme}

Problem (29)-(32) is in general NP-hard for the optimal solution. To obtain an insight into the problem, we first investigate the properties of the case with a given subcarrier assignment, which are summarized in the following theorem.

Theorem I. For any fixed subcarrier assignment indicator matrix $\rho$ and its corresponding subcarrier assignment sets $\mathcal{S}_{k}(\forall k \in \mathcal{K})$, the maximum achievable $R E$ at a certain total transmit power, $P_{T}$, namely,

$$
\bar{\lambda}_{R E}\left(P_{T}\right) \triangleq \max _{p_{k, n} \geq 0} \frac{\sum_{k \in \mathcal{K}} \sum_{n \in \mathcal{S}_{k}} r_{k, n}}{\zeta P_{T}+P_{C}}\left(1+\beta \frac{\eta_{P}}{\eta_{W}}\right)
$$


subject to

$$
\begin{gathered}
\sum_{n \in \mathcal{S}_{k}} r_{k, n} \geq \gamma_{k}, \forall k \in \mathcal{K} \\
\sum_{k \in \mathcal{K}} \sum_{n \in \mathcal{S}_{k}} p_{k, n}=P_{T} \\
P_{T} \leq P_{\max }
\end{gathered}
$$

has the following properties:

(i) $\bar{\lambda}_{R E}\left(P_{T}\right)$ is continuously differentiable and quasiconcave in $P_{T}$,

(ii) The derivative of $R E$ satisfies

$$
\frac{d \bar{\lambda}_{R E}\left(P_{T}\right)}{d P_{T}}=\frac{\left(\beta \frac{\eta_{P}}{\eta_{W}}+1\right) \frac{d \bar{R}\left(P_{T}\right)}{P_{T}}-\zeta \bar{\lambda}_{E E}\left(P_{T}\right)}{\zeta P_{T}+P_{C}}
$$

where

$$
\begin{aligned}
\bar{\lambda}_{E E}\left(P_{T}\right) & =\frac{\bar{R}\left(P_{T}\right)}{\zeta P_{T}+P_{C}} \\
\bar{R}\left(P_{T}\right) \triangleq \max _{p_{k, n} \geq 0} R\left(P_{T}\right) & =\max _{p_{k, n} \geq 0} \sum_{k \in \mathcal{K}} \sum_{n \in \mathcal{S}_{k}} r_{k, n}
\end{aligned}
$$

is the maximum sum rate under constraints (34)-(36), and its derivative satisfies

$$
\frac{d \bar{R}\left(P_{T}\right)}{P_{T}}=\max _{k \in \mathcal{K}, n \in \mathcal{S}_{k}} \frac{W_{C} g_{k, n} \log _{2} e}{1+\bar{p}_{k, n} g_{k, n}}
$$

where $g_{k, n} \triangleq \frac{\left|H_{k, n}\right|^{2}}{N_{0} W_{C}}$ is the channel gain to noise ratio of the $k^{\text {th }} U E$ on the $n^{\text {th }}$ subcarrier and $\bar{p}_{k, n}\left(n \in \mathcal{S}_{k}\right)$ is the optimal power on the $n^{\text {th }}$ subcarrier for achieving $\bar{R}\left(P_{T}\right)$.

Proof: see Appendix A.

Since transmission power $P_{T}$ and subcarrier assignment indicator matrix $\rho$ are fixed in this case, $\operatorname{RE} \bar{\lambda}_{R E}$ can be rewritten as follows,

$$
\bar{\lambda}_{R E}\left(P_{T}\right)=\max _{p_{k, n} \geq 0} R\left(\frac{1}{P}+\beta \frac{W_{t o t}}{W P_{t o t}}\right)=\omega \bar{R}\left(P_{T}\right),
$$

where $\omega \triangleq \frac{1}{P}+\beta \frac{W_{t o t}}{W P_{t o t}}$. Therefore, our next step is to find out the optimal power allocation scheme to maximize (41). Similar to [16], the optimal power can be calculated using the following water-filling scheme

$$
\begin{gathered}
\tilde{p}_{k, n}=\left(\mu_{k}-\frac{1}{g_{k, n}}\right)^{+}, \forall n \in \mathcal{S}_{k}, \\
\sum_{n \in \mathcal{S}_{k}, \tilde{p}_{k, n}>0} W_{C} \log _{2}\left(\mu_{k} g_{k, n}\right)=\gamma_{k}, \\
\bar{p}_{k, n}=\tilde{p}_{k, n}+\left(\mu-\frac{1}{g_{k, n}}-\tilde{p}_{k, n}\right)^{+},
\end{gathered}
$$

$\sum_{k \in \mathcal{K}} \sum_{n \in\left\{\mathcal{S}_{k} \mid \bar{p}_{k, n}>\tilde{p}_{k, n}\right\}}\left(\mu-\frac{1}{g_{k, n}}-\tilde{p}_{k, n}\right)=P_{T}-\sum_{k \in \mathcal{K}} \sum_{n \in \mathcal{S}_{k}} \tilde{p}_{k, n}$,

where $\mu_{k}$ and $\mu$ are intermediate variables. The basic idea of the power allocation process contains two steps. The first step is to allocate power to make each UE merely satisfy its rate requirement. The power used in this step is $P_{S}=\sum_{k \in \mathcal{K}} \sum_{n \in \mathcal{S}_{k}} \tilde{p}_{k, n}$. The second step is to allocate the remaining power to the subcarriers that can further maximize the sum rate. This scheme can be straightforwardly realized

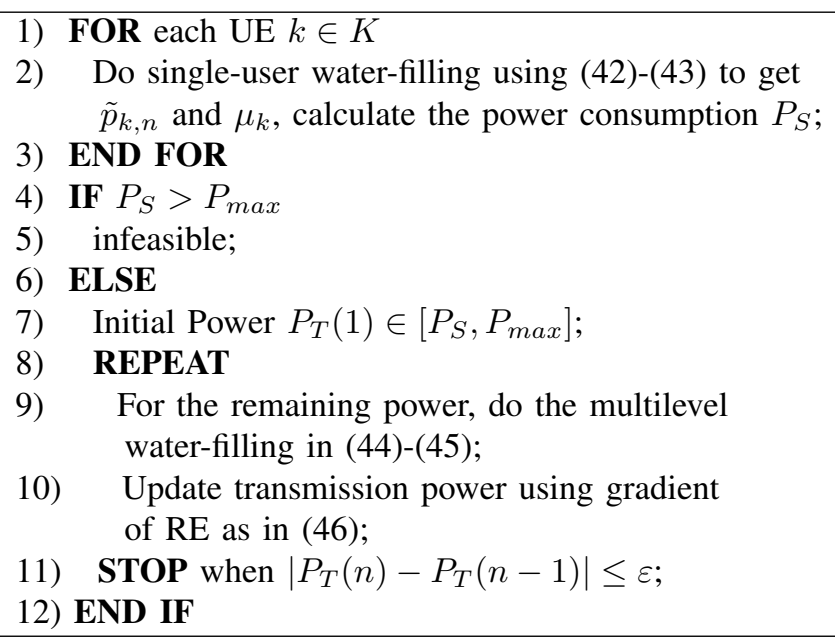

TABLE I

GRADIENT-BASED OPTIMAL POWER ADAPTATION SCHEME

by the method of Lagrange multiplier and the derivations of (42)-(45).

There exists a unique global maximum for any quasiconcave function. As a result, Property (i) in Theorem I guarantees the existence and uniqueness of the global maximum and reveals the differentiability of $\bar{\lambda}_{R E}\left(P_{T}\right)$. Furthermore, $\bar{\lambda}_{R E}\left(P_{T}\right)$ either strictly decreases or first increases and then strictly decreases with $P_{T}$ starting from $\sum_{k \in \mathcal{K}} R_{k}^{-1}\left(\mathcal{S}_{k}, \gamma_{k}\right)$ which is the minimum transmit power required for realizing aggregate rate $\gamma_{k}$ over subcarrier set for all the UEs. Property (ii) further indicates that the maximum point is always achieved at a finite transmit power. It connects the sign of the first derivative with the relative size of RE and the scaled reciprocal of the waterfilling level. For the fixed subcarrier assignment, Theorem I indicates that with an initial power value $P_{T}(1)$, the optimal power allocation scheme for (33)-(36) can be easily obtained by a derivative-assisted gradient scheme which is based on the single-UE water-filling in (42)-(43), and the multilevel waterfilling in (44)-(45). The algorithm is called gradient-based power adaptation. The power is updated using the gradient of RE as follows,

$$
P_{T}(n)=P_{T}(n-1)+t \times \frac{d \bar{\lambda}_{R E}\left(P_{T}\right)}{d P_{T}}
$$

where $t$ is the step size. Since $\bar{\lambda}_{R E}\left(P_{T}\right)$ either strictly decreases or first increases and then strictly decreases with $P_{T}$, the proposed approach will end with either convergence or $P_{0}=\sum_{k \in \mathcal{K}} R_{k}^{-1}\left(\mathcal{S}_{k}, \gamma_{k}\right)$ if $\bar{\lambda}_{R E}\left(P_{T}\right)$ is monotonically decreasing in $\left[P_{0}, P_{\max }\right]$ and $P_{\max }$ if $\bar{\lambda}_{R E}\left(P_{T}\right)$ is monotonically increasing in $\left[P_{0}, P_{\max }\right]$. The algorithm is detailed in Table I.

The optimal solution to (29)-(32) can be obtained by applying the gradient-based power adaptation algorithm to every feasible subcarrier assignment and then choose the one with the maximum RE. However, the complexity is extremely high and makes it prohibitive for practical scenarios.

\section{B. Upper Bound Near Optimal Resource Allocation Scheme}

To facilitate practical implementation of the optimal resource efficient design, we will first exploit and prove the quasiconcave relation between an upper bound 
$\max _{\boldsymbol{\rho}, p_{k, n}} \tilde{\lambda}_{R E}\left(P_{T}\right)$ and total transmit power, $P_{T}$. Different from previous definition where $\rho_{k, n} \in\{1,0\}$ indicates whether or not the $n^{t h}$ subcarrier is assigned to the $k^{t h} \mathrm{UE}$, we need to relax $\rho_{k, n}$ as follows

$$
0 \leq \rho_{k, n} \leq 1 \text { and } Q=\sum_{k \in \mathcal{K}} \sum_{n \in \mathcal{N}} \rho_{k, n} .
$$

The fractional $\rho_{k, n}$ can be either interpreted as time domain sharing of subcarriers [26], [27]. Also, the maximum achievable data rate of the $k^{t h} \mathrm{UE}$ on the $n^{\text {th }}$ subcarrier is accordingly

$$
\tilde{r}_{k, n}=W_{C} \log _{2}\left(1+\frac{p_{k, n}\left|H_{k, n}\right|^{2}}{\rho_{k, n} N_{0} W_{C}}\right)
$$

Clearly, by relaxing $\rho_{k, n}$ as (47) and reformulating the maximum achievable data rate $\tilde{r}_{k, n}$ in (48), the RE optimization problem always yields an upper bound on the RE of (29)-(32), i.e., $\tilde{\lambda}_{R E}^{U B} \geq \bar{\lambda}_{R E}$, although it does not necessarily guarantee a solution where $\rho_{k, n}$ is either 0 or 1 .

Theorem II. The upper bound on the maximum achievable $R E, \max _{\boldsymbol{\rho}, p_{k, n}} \tilde{\lambda}_{R E}\left(P_{T}\right)$, at a given number of subcarriers $Q$ and a certain total transmit power, $P_{T}$, namely

$$
\tilde{\lambda}_{R E}^{U B}\left(P_{T}\right) \triangleq \max _{\boldsymbol{\rho}, p_{k, n} \geq 0} \frac{\sum_{k \in \mathcal{K}} \sum_{n \in \mathcal{S}_{k}} \rho_{k, n} \tilde{r}_{k, n}}{\zeta P_{T}+P_{C}}\left(1+\beta \frac{\eta_{P}}{\eta_{W}}\right)
$$

subject to

$$
\begin{gathered}
\sum_{n \in \mathcal{N}} \rho_{k, n} \tilde{r}_{k, n} \geq \gamma_{k}, \forall k \in \mathcal{K} \\
\sum_{k \in \mathcal{K}} \sum_{n \in \mathcal{S}_{k}} p_{k, n}=P_{T} \\
\sum_{k \in \mathcal{K}} \sum_{n \in \mathcal{N}} \rho_{k, n}=Q
\end{gathered}
$$

has the following properties:

(i) $\tilde{\lambda}_{R E}^{U B}\left(P_{T}\right)$ is continuously differentiable and quasiconcave in $P_{T}$,

(ii) The derivative of upper bound RE satisfies

$$
\frac{d \tilde{\lambda}_{R E}^{U B}\left(P_{T}\right)}{d P_{T}}=\frac{\left(\beta \frac{\eta_{P}}{\eta_{W}}+1\right) \frac{d \tilde{R}\left(P_{T}\right)}{P_{T}}-\zeta \tilde{\lambda}_{E E}\left(P_{T}\right)}{\zeta P_{T}+P_{C}}
$$

where

$$
\begin{aligned}
\tilde{\lambda}_{E E}\left(P_{T}\right) & =\frac{\tilde{R}\left(P_{T}\right)}{\zeta P_{T}+P_{C}} \\
\tilde{R}\left(P_{T}\right) \triangleq \max _{\boldsymbol{\rho}, p_{k, n} \geq 0} R\left(P_{T}\right) & =\max _{\boldsymbol{\rho}, p_{k, n} \geq 0} \sum_{k \in \mathcal{K}} \sum_{n \in \mathcal{N}} \rho_{k, n} \tilde{r}_{k, n}
\end{aligned}
$$

is the maximum sum rate under constraints (34)-(36), and its derivative satisfies

$$
\frac{d \tilde{R}\left(P_{T}\right)}{P_{T}}=\max _{k \in \mathcal{K}, n \in \mathcal{N}} \frac{W_{C} \tilde{\rho}_{k, n} g_{k, n} \log _{2} e}{1+\tilde{\rho}_{k, n} \tilde{p}_{k, n} g_{k, n}}
$$

where $\tilde{\rho}_{k, n}$ and $\tilde{p}_{k, n}$ is the optimal subcarrier and power allocation for achieving $\tilde{R}\left(P_{T}\right)$.

Proof: The proof is similar to that of Theorem $I$ in Appendix A but with the inclusion of time sharing factor $\rho$. Therefore the proof is ignore here.
More importantly, as a result of the quasiconcavity, problem (49)-(52) can be decomposed into two layers and solved iteratively by the joint inner- and outer-layer optimization as follows:

(i) Inner-layer: For a given transmit power, $P_{T} \leq P_{\max }$, finds the maximum $\operatorname{RE} \tilde{\lambda}_{R E}^{U B}\left(P_{T}\right)$, and the gradient of $\operatorname{RE} \frac{d \tilde{R}\left(P_{T}\right)}{P_{T}}$. (ii) Outer-layer: Finds the optimal RE, $\lambda_{R E}^{o p t}$, via a gradientbased power adaption algorithm in accordance with Theorem II.

The key lies in the inner-layer algorithm that finds $\tilde{\lambda}_{R E}^{U B}\left(P_{T}\right)$ and the gradient of $\operatorname{RE} \frac{d \tilde{R}\left(P_{T}\right)}{P_{T}}$. For a given total transmit power $P_{T}$, the inner-layer subproblem to find $\tilde{\lambda}_{R E}^{U B}\left(P_{T}\right)$ is equivalent to maximizing the constrained sum rate, which is proved to be strictly and jointly concave in $\rho_{k, n}$ and $p_{k, n}$ [28]. The constrained sum rate maximization is in the standard form of a convex programming problem that can be solved by standard numerical methods such as the interior-point method [29]. When the optimal subcarrier assignment $\rho_{k, n}$, and power allocation $p_{k, n}$, for the constrained sum rate maximization problem are obtained, the gradient of $\mathrm{RE} \frac{d \tilde{R}\left(P_{T}\right)}{P_{T}}$ can be readily determined following Property (ii) in Theorem II. Hence, problem (49)-(52) can be successfully solved by the aforementioned joint inner- and outer-layer optimization.

When $\tilde{\lambda}_{R E}^{U B} \mathrm{EE}$ is found, the corresponding optimal $\rho_{k, n}$ are not ensured to be either 0 or 1 . To get a feasible solution to the original downlink RE maximization problem, we need to round the possibly fractional $\rho_{k, n}$ to 0 or 1 and then perform the gradient-based algorithm to get the maximum RE for the round-off $\rho_{k, n}$. Such manipulations may not result in the optimal solution to (49)-(52). Nevertheless, this is rarely a problem when the number of subcarriers is large comparing to the number of UEs and in this case $\tilde{\lambda}_{R E}^{U B}$ RE is quite close to $\bar{\lambda}_{R E}$. In fact, the optimal $\rho_{k, n}$ for the constrained sum rate maximization problem mostly tend to be either 0 or 1 when $K \ll N$ [28]. On the other hand, such fine tightness of the RE upper bound and the fact that the optimal $\rho_{k, n}$ are almost either 0 or 1 implicitly enable the use of the original sum rate, instead of the constrained sum rate for maximization in the inner-layer optimization with an expectation of good performance. This enables us to precisely solve the original problem (49)-(52) by the joint inner- and outer-layer optimization framework with the constrained sum rate maximization as the inner-layer subproblem.

The solution to (29)-(32) can be obtained by applying the interior point method to all different number of subcarriers, i.e., from $Q=K$ to $Q=N$, and then choose the one with the maximum RE.

\section{Suboptimal Low Complexity Scheme}

Although convex programming is numerically stable, its computational complexity depends on the number of optimizing variables, which can be large if the number of subcarriers or the number of UEs is large. As for the optimal solution, we need to check all possible number of active subcarriers and apply water-filling algorithm and gradient updated scheme for each case to find the optimal power allocation. The complexity of this scheme is comparably high. Here, we will explore 
the property of proposed optimization problem in (29)-(32) using uniform power allocation scheme and propose a novel low-complexity suboptimal algorithm which is based on the following theorems.

Theorem III. For a fixed transmission power $P_{T}$, with uniform power allocation scheme, the maximum achievable $R E$ at a certain bandwidth, W, namely

$$
\check{\lambda}_{R E}(W) \triangleq \max _{\rho} \frac{\sum_{k \in \mathcal{K}} \sum_{n \in \mathcal{N}} \rho_{k, n} r_{k, n}}{\zeta P_{T}+P_{C}}\left(1+\beta \frac{\eta_{P}}{\eta_{W}}\right)
$$

subject to

$$
\begin{gathered}
\sum_{n \in \mathcal{N}} \rho_{k, n} r_{k, n} \geq \gamma_{k}, \forall k \in \mathcal{K} \\
p_{k, n}=\rho_{k, n} \frac{P_{T}}{Q}, \forall k \in \mathcal{K}, \forall n \in \mathcal{N} \\
\sum_{k \in \mathcal{K}} \sum_{n \in \mathcal{N}} \rho_{k, n} W_{C}=W
\end{gathered}
$$

either strictly decreases or first strictly increases and then strictly decreases with $Q$.

Proof: See proof in Appendix B.

Theorem IV. For any fixed subcarrier assignment indicator matrix $\rho$ and its corresponding subcarrier assignment sets $\mathcal{S}_{k}(\forall k \in \mathcal{K})$, with uniform power allocation scheme, the maximum achievable RE at a certain total transmit power, $P_{T}$, namely,

$$
\hat{\lambda}_{R E}\left(P_{T}\right) \triangleq \max _{p_{k, n} \geq 0} \frac{\sum_{k \in \mathcal{K}} \sum_{n \in \mathcal{S}_{k}} r_{k, n}}{\zeta P_{T}+P_{C}}\left(1+\beta \frac{\eta_{P}}{\eta_{W}}\right)
$$

subject to

$$
\begin{gathered}
\sum_{n \in \mathcal{S}_{k}} \rho_{k, n} r_{k, n} \geq \gamma_{k}, \forall k \in \mathcal{K} \\
p_{k, n}=\frac{P_{T}}{Q}, \forall k \in \mathcal{K}, \forall n \in \mathcal{S}_{k}
\end{gathered}
$$

is continuously differentiable and quasiconcave in $P_{T}$, and has a derivative that satisfies $\frac{d \hat{\lambda}_{R E}\left(P_{T}\right)}{d P_{T}}=$ $\frac{\left(\beta \frac{\eta_{P}}{\eta_{W}}+1\right) \sum_{k \in \mathcal{K}} \sum_{n \in \mathcal{S}_{k}} \frac{\frac{W_{C} \log _{2} e}{g_{k, n}}+P_{T}}{g} \zeta \hat{\lambda}_{E E}\left(P_{T}\right)}{\zeta P_{T}+P_{C_{P}}}$, where $\hat{\lambda}_{E E}\left(P_{T}\right)=$ $\frac{\sum_{k \in \mathcal{K}} \sum_{n \in \mathcal{S}_{k}} W_{C} \log _{2}\left(1+\frac{P_{T}}{Q} g_{k, n}\right)}{\zeta P_{T}+P_{C}}$.

Proof: See proof in Appendix C.

The basic idea of the proposed suboptimal scheme is solving the problem in (57)-(60) using water-filling scheme similar to (42)-(45) to find out how much bandwidth is suitable for the network with initial power setting $P_{T}$. Since Theorem III reveals that $\check{\lambda}_{R E}(W)$ either strictly decreases or first strictly increases and then strictly decreases with $Q$, we can determine the optimal number of subcarriers by comparing two consecutive RE values (i.e., $\check{\lambda}_{R E}\left(Q \times W_{C}\right)$ and $\left.\check{\lambda}_{R E}\left((Q+1) \times W_{C}\right)\right)$. If $\check{\lambda}_{R E}\left(Q \times W_{C}\right)<\check{\lambda}_{R E}\left((Q+1) \times W_{C}\right)$, we treat the number of subcarriers used in the previous iteration as the target subcarriers. Otherwise, we keep increasing the number of active subcarriers. With the defined bandwidth, i.e., number of subcarriers, and the gradient of RE we find in Theorem IV ( $\left.\frac{d \hat{\lambda}_{R E}\left(P_{T}\right)}{d P_{T}}\right)$, we solve the problem in (61)-(63) to find out the optimal transmission power. We then
1) Initial transmission power $P_{T}(1), \varepsilon=0.01, n=1$;

\section{2) REPEAT}

3) Initial number of subcarrier $Q=K, l=1$, exit $=0$;

4) WHILE exit $=0$

5) Solve the problem in (57)-(60) using uniform power allocation scheme and store the value of RE $\check{\lambda}_{R E}^{(l)}$;

6) IF $\check{\lambda}_{R E}^{(l)}>\check{\lambda}_{R E}^{(l-1)}$

7) $Q=Q+1, l=l+1$;

8) ELSE

9) $\quad$ exit $=1 ; L(n)=Q-1$;

\section{0) END IF}

11) END WHILE

12) Update $P_{T}(n)$ using (64); $n=n+1$;

13)STOP when $L(n)=L(n-1)$ and $\left|P_{T}(n)-P(n-1)\right| \leq \varepsilon ;$

14)Initial transmission power $P_{T}(n)$ and number of subcarrier $Q=L(n)$;

15)Do single-user water-filling using (42)-(43);

16)For the remaining power, do the multilevel water-filling in (44)-(45).

TABLE II

LOW COMPLEXITY SUBOPTIMAL SCHEME

update the transmission power $P_{T}$ to solve the problem in (57)-(60) again. This procedure is repeated until convergence (both subcarriers and transmission power). Details of the proposed suboptimal scheme is discussed in the following.

We first initiate a transmission power $P_{T}(1)$ and an initial number of subcarriers $Q=K$. The reason for choosing the initial number of subcarriers as $K$ is that there exists $K$ users in the network, we should have at least $K$ subcarriers to satisfy each user's minimum data rate requirements. Using uniform power allocation, we store the current RE value $\check{\lambda}_{R E}^{(l)}$ in the buffer. Then we increment the number of subcarriers as $Q=Q+1$ and calculate the RE value $\check{\lambda}_{R E}^{(l+1)}$. If the current $\mathrm{RE}$ is larger than the previous one, we further increase the number of subcarriers as $Q=Q+1$. Otherwise, we treat the number of subcarriers used in the previous iteration as the target subcarriers, we store this result in the buffer as $L(n)=Q-1$. This procedure is referred to as subcarrier selection. Then we need to find out the suitable transmission power $P_{T}$ based on the chosen subcarriers. We can update the transmission power $P_{T}$ using the gradient $\frac{d \hat{\lambda}_{R E}\left(P_{T}\right)}{d P_{T}}$ from Theorem IV as follows

$$
P_{T}(n)=P_{T}(n-1)+t \times \frac{d \hat{\lambda}_{R E}\left(P_{T}\right)}{d P_{T}}
$$

where $t$ is the step size. This power updating procedure is repeated until its convergence (i.e., $\left|P_{T}(n)-P_{T}(n-1)\right| \leq \varepsilon$ ). Once we have the updated transmission power $P_{T}$, we use it as the updated initial transmission power for the next iteration, and run the subcarrier defining method again. The overall procedure is repeated until its convergence (both subcarriers and transmission power are fixed). Finally, based on the chosen subcarriers and transmission power, we apply water-filling algorithm in (42)-(45) to find out the optimal power allocation. The algorithm is presented in Table II.

In Table III, the complexity of the aforementioned optimal, 


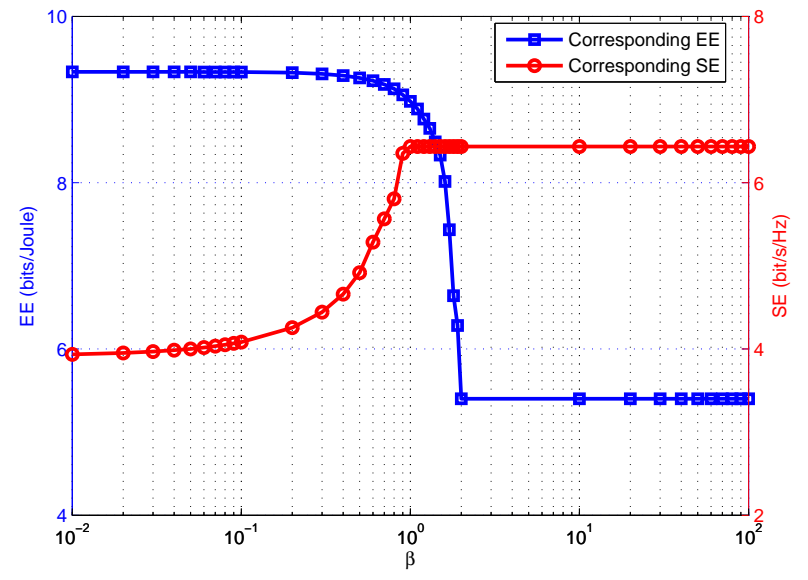

Fig. 2. Impact of weighted factor $\beta$ to the corresponding $\mathrm{EE}$ and $\mathrm{SE}$ $\left(P_{\max }=2, P_{C}=1, K=4, N=16, W_{C}=1 \mathrm{~Hz}\right.$ and $\gamma_{k}=4$ $\mathrm{bit} / \mathrm{s} / \mathrm{Hz})$.

near-optimal upper bound, and low-complexity alternative is listed for comparison. We calculate the complexity based on the number of floating point.

\begin{tabular}{|c|c|}
\hline Algorithm & Complexity \\
\hline Optimal scheme & $\mathcal{O}\left(L K^{K} \frac{1-K^{(N-K+1)}}{1-K}\right)$ \\
\hline Upper bound scheme & $\mathcal{O}\left(\frac{1}{\delta^{2}} L K(N-K+1) \frac{K+N}{2}\right)$ \\
\hline Suboptimal scheme & $\mathcal{O}\left(L \frac{N-K+1}{2}\right)$ \\
\hline
\end{tabular}

TABLE III

COMPLEXITY COMPARISON FOR THE PROPOSED ALGORITHMS

\section{Simulation RESUlts}

In this section, we present simulation results to verify the benefit of the proposed RE and the proposed allocation algorithm. In our simulation, the circuit power is normalized to $1 \mathrm{~W}$ while the drain efficiency of the power amplifier is $38 \%$ for our simulation [16]. These parameters are chosen to demonstrate the concept of RE, and can easily be modified to any other values for different scenarios. The channels are frequency selective Rayleigh faded with zero mean and unit variance. To provide different service priorities and guaranteed QoS for each UE, we consider the generalized RE under the minimum rate requirements $\gamma_{k}$ of $4 \mathrm{bit} / \mathrm{s} / \mathrm{Hz}$.

In the first simulation, we investigate the impact of weighted factor $\beta$ to the corresponding EE and SE. Fig. 2 plots the weighted factor $\beta$ versus the corresponding EE and SE. As can be seen from the figure, the corresponding EE decreases with increasing $\beta$ while the corresponding SE increases with increasing $\beta$. This is because increasing $\beta$ leads to more weight putting on SE and hence more resources are allocated for maximizing SE. Furthermore, the corresponding EE and $\mathrm{SE}$ remains the same when weighted factor $\beta$ is either small or large. As explained before, when $\beta$ is small, $\mathrm{RE}$ will focus on optimizing EE, but will optimize SE when $\beta$ is large. As shown in Fig. 3, when $\beta$ is in the range from 0.1 to 1 , the corresponding EE decreases gradually with increasing $\beta$ while the corresponding SE increases gradually with increasing $\beta$. In particular, when $\beta=1$, both corresponding $\mathrm{EE}$ and $\mathrm{SE}$ are

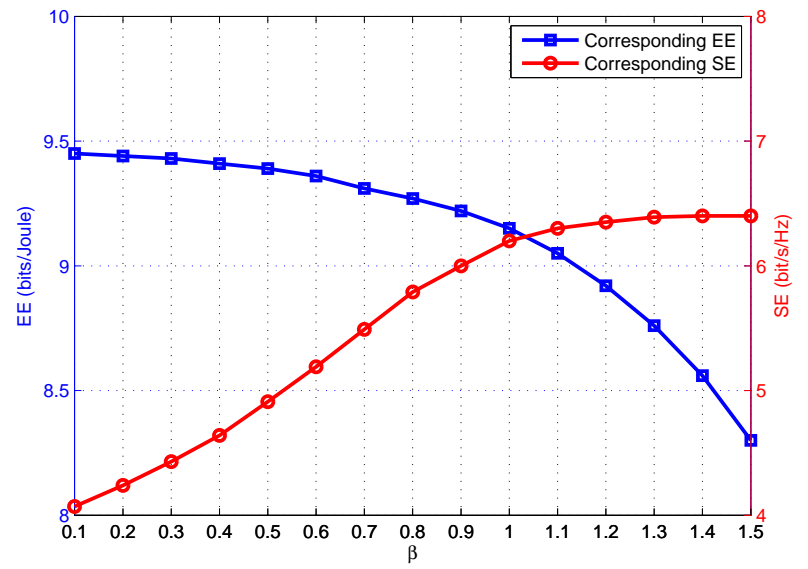

Fig. 3. Impact of weighted factor $\beta$ to the corresponding $\mathrm{EE}$ and $\mathrm{SE}$ $\left(P_{\max }=2, P_{C}=1, K=4, N=16, W_{C}=1 \mathrm{~Hz}\right.$ and $\gamma_{k}=4$ $\mathrm{bit} / \mathrm{s} / \mathrm{Hz})$.

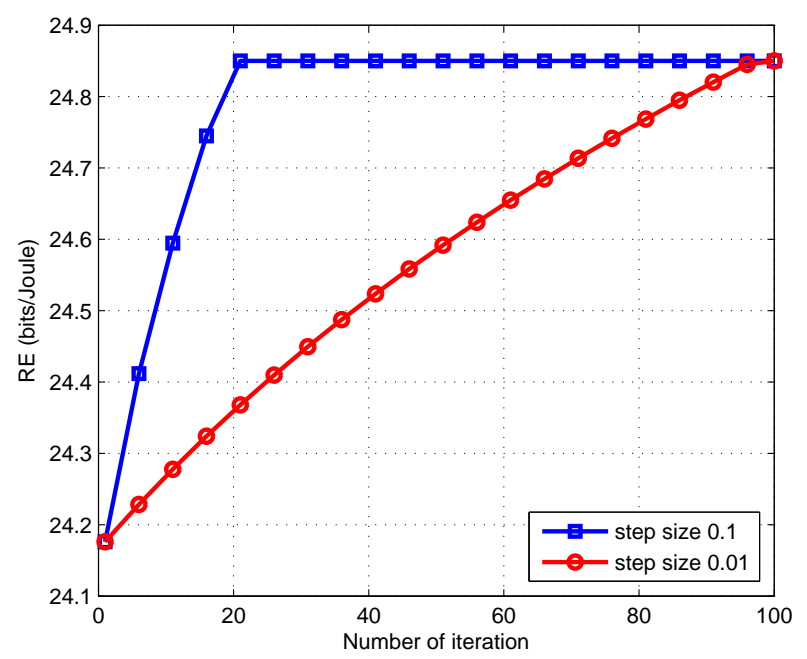

Fig. 4. The convergence behavior of the proposed gradient-based optimal power adaptation scheme $\left(P_{\max }=2, P_{C}=1, K=4, N=16, W_{C}=1\right.$ $\mathrm{Hz}$ and $\gamma_{k}=4 \mathrm{bit} / \mathrm{s} / \mathrm{Hz}$ ).

close to the maximum $\mathrm{EE}$ (when $\beta=0$ ) and maximum SE (when $\beta=\infty$ ). The amount of degradation is only $4 \%$ on $\mathrm{EE}$ and $2 \%$ on SE. This result justify the proposed use of $\frac{W_{t o t}}{P_{t o t}}$ as the normalization factor in the RE definition. As a result, we set $\beta$ to unity in the following simulation sections.

We then evaluate the performance of all the proposed schemes. We first show a figure for the proposed gradientbased optimal power adaptation scheme which illustrates how the system performance improves with the number of iterations. Fig. 4 plots resource efficiency versus the number of iterations of the proposed gradient-based optimal power adaptation scheme for step sizes $t=0.1$ and $t=0.01$. As can be seen from the figure, the step size affects the accuracy and convergence speed of the algorithm. Nevertheless, both step size converge to the same optimal point. We then compare the proposed suboptimal low complexity scheme with the gradient-based optimal power adaptation scheme, near optimal upper bound approach and the suboptimal resource allocation scheme proposed in [16]. The average channel gain to noise 


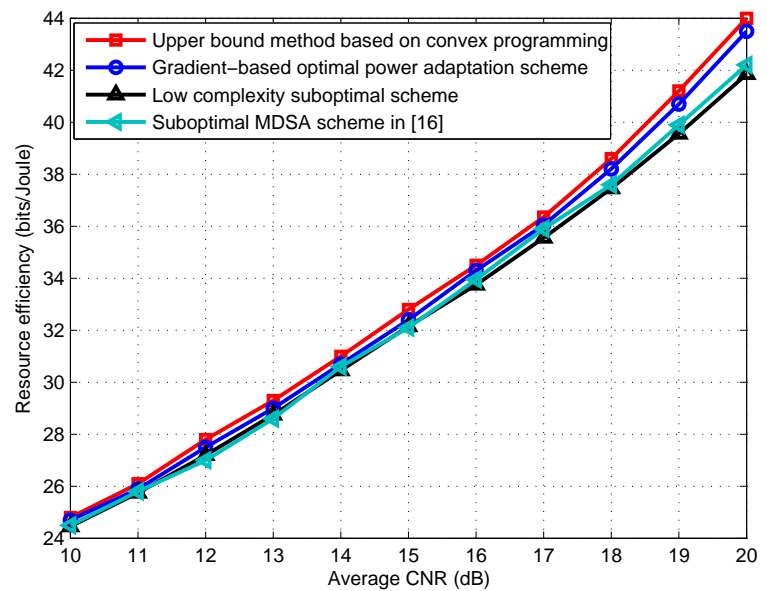

Fig. 5. Comparison of suboptimal low complexity scheme with gradientbased optimal power adaptation scheme and near optimal upper bound scheme. $\left(P_{\max }=2, P_{C}=1, K=4, N=16, W_{C}=1 \mathrm{~Hz}\right.$ and $\left.\gamma_{k}=4 \mathrm{bit} / \mathrm{s} / \mathrm{Hz}\right)$

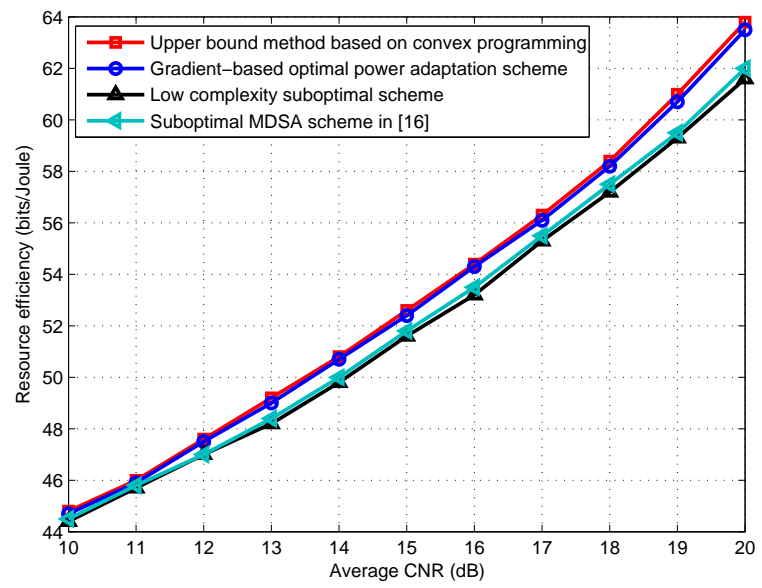

Fig. 6. Comparison of suboptimal low complexity scheme with gradientbased optimal power adaptation scheme and near optimal upper bound scheme. $\left(P_{\max }=30, P_{C}=15, K=50, N=256, W_{C}=1 \mathrm{~Hz}\right.$ and $\left.\gamma_{k}=4 \mathrm{bit} / \mathrm{s} / \mathrm{Hz}\right)$

ratio is varied from $10 \mathrm{~dB}$ to $20 \mathrm{~dB}$. The total number of subcarriers $N$ and UEs $K$ in our network is first set to 16 and 4 respectively. Fig. 5 shows that the RE achieved by the near optimal upper bound method is close to that of the gradient-based optimal power adaptation scheme. Specifically, the proposed suboptimal scheme results in an RE that is at least $95 \%$ of the optimal RE. Furthermore, the proposed suboptimal scheme is very close to the suboptimal resource allocation scheme proposed in [16] but with much lower complexity. Hence, we can employ the proposed suboptimal scheme to lower the system's complexity while achieving near optimal performance. We then increase the total number of subcarriers $N$ and UEs $K$ to 256 and 50 respectively. As can be seen from Fig. 6, the trend is similar with large number of subcarriers, whereby the RE achieved by the near optimal upper bound method is almost the same as that of the gradientbased optimal power adaptation scheme. The proposed low complexity suboptimal scheme is also very close to suboptimal

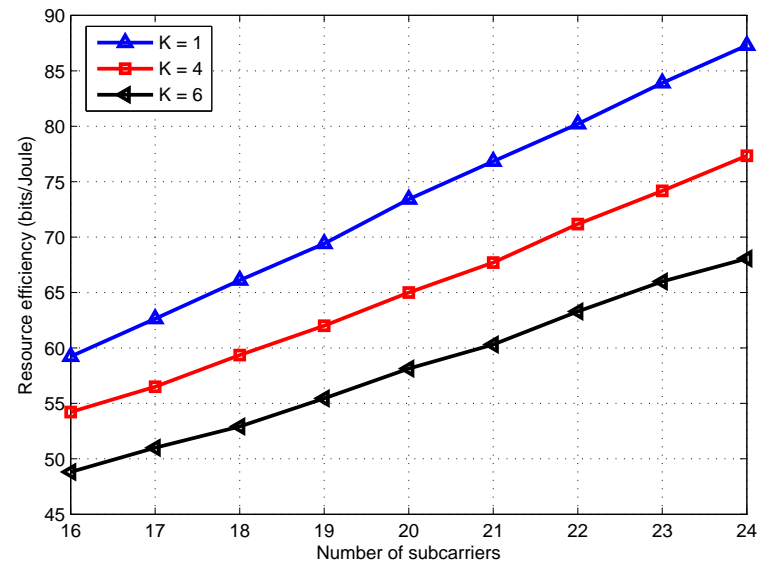

Fig. 7. Comparison of the maximum achievable RE obtained by gradientbased optimal power adaptation scheme for different number of subcarriers. $\left(P_{\max }=1, P_{C}=1, K=4, W_{C}=1 \mathrm{~Hz}\right.$ and $\left.\gamma_{k}=4 \mathrm{bit} / \mathrm{s} / \mathrm{Hz}\right)$

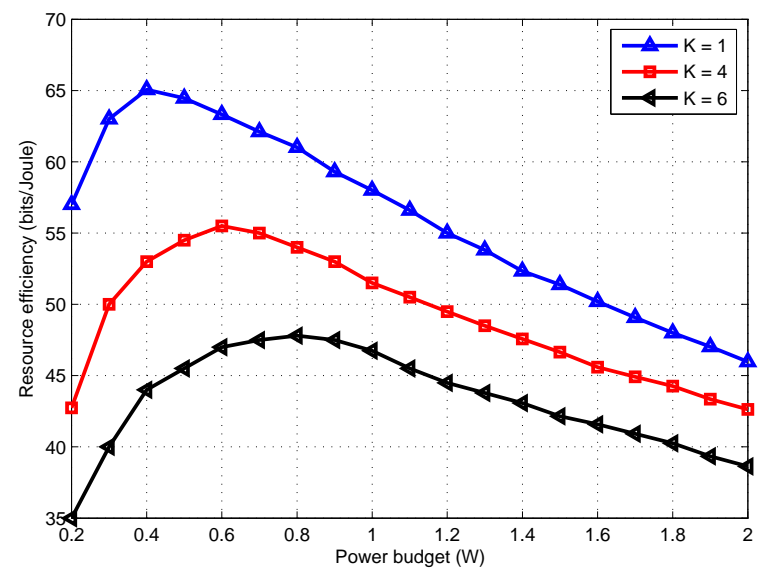

Fig. 8. Comparison of the maximum achievable RE obtained by gradientbased optimal power adaptation scheme for different transmission power budget. $\left(P_{C}=1, K=4, N=16, W_{C}=1 \mathrm{~Hz}\right.$ and $\left.\gamma_{k}=4 \mathrm{bit} / \mathrm{s} / \mathrm{Hz}\right)$

resource allocation scheme proposed in [16].

In the next simulation, we analyse the impact of different number of subcarriers $N$ to the maximum achievable RE. Fig. 7 shows the comparison of maximum achievable RE obtained by the proposed gradient-based optimal power adaptation scheme for different number of subcarriers from 16 to 24 . As expected, the maximum achievable RE increases as the number of subcarriers increases. This is because when the number of subcarriers increases, the network has more freedom in terms of choosing subcarriers subset, and hence can have better resource utilization. Thus this increases the maximum achievable RE. Furthermore, the trends of RE are similar for different number of UEs $(K)$. Interestingly, Fig. 7 reveals that a higher number of users leads to lower achievable RE. Although more users in the network provide user diversity, satisfying each user's minimum rate requirements $\left(\gamma_{k}\right.$ of 4 $\mathrm{bit} / \mathrm{s} / \mathrm{Hz}$ in the simulation) leads to inefficient use of resources hence lowering the achievable RE.

We analyse the impact of different transmission power budget $P_{\max }$ to the maximum achievable RE. The number 


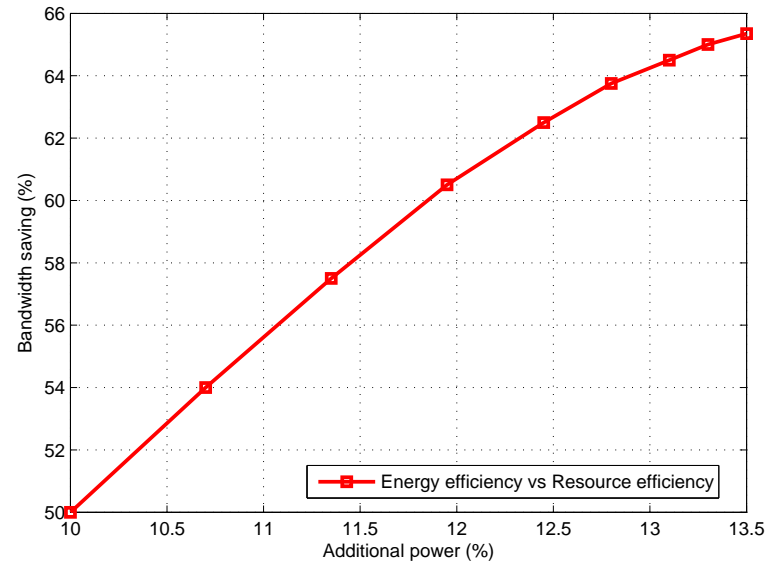

Fig. 9. Energy efficiency versus resource efficiency for tradeoff between extra power and bandwidth saving. $\left(P_{\max }=1, P_{C}=1, K=4, N=16\right.$ and $W_{C}=1 \mathrm{~Hz}$

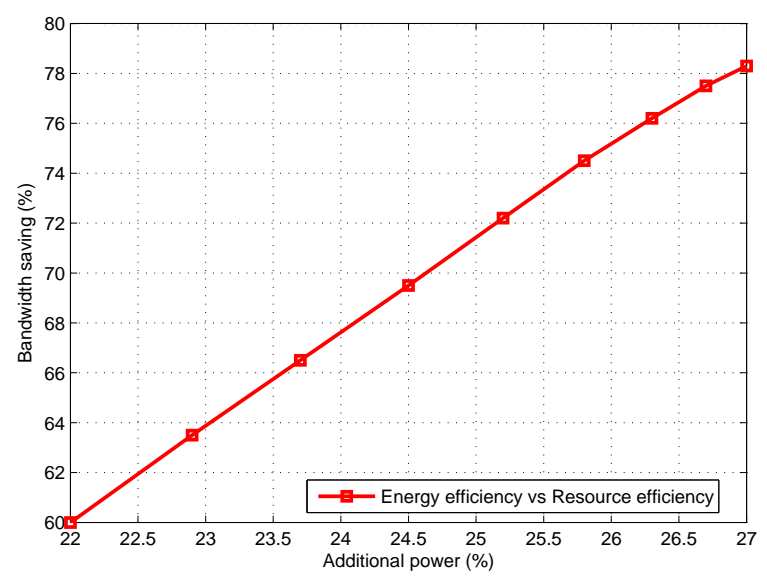

Fig. 10. Energy efficiency versus resource efficiency for tradeoff between extra power and bandwidth saving. $\left(P_{\max }=1, P_{C}=1, K=50, N=256\right.$ and $W_{C}=1 \mathrm{~Hz}$ )

of subcarriers is fixed to 16. Fig. 8 shows the comparison of the maximum achievable RE obtained by the proposed gradient-based optimal power adaptation scheme for different transmission power budget from $0.2 \mathrm{~W}$ to $2 \mathrm{~W}$. As can be seen from Fig. 8, the maximum achievable RE first increases then decreases with a turning point around $0.4 \mathrm{~W}$ for $K=$ 1 and $0.8 \mathrm{~W}$ for $K=6$. This is because RE is defined as $\frac{R}{P}\left(1+\frac{\eta_{P}}{\eta_{W}}\right)$, where $\eta_{P} \triangleq \frac{P}{\zeta P_{\max }+P_{C}}$. When $P_{\max }$ is comparably low (i.e., $P_{\max }<0.8 W$ for $K=6$ ), the maximum achievable RE increases when the transmission power budget $P_{\max }$ increases. However, when $P_{\max }$ is comparably high, the maximum achievable RE decreases when the transmission power budget $P_{\max }$ increases. This is very important for green cellular networks as we can save much power by lowering the transmission power budget.

We finally show the performance of the RE based approach compared to conventional $\mathrm{EE}$ and $\mathrm{SE}$ ones in terms of resource saving by varying the minimum rate requirements from 1 bit/s/Hz to $5 \mathrm{bit} / \mathrm{s} / \mathrm{Hz}$ (at 0.5 interval). Fig. 9 and Fig. 10 show EE versus RE for tradeoff between extra power and

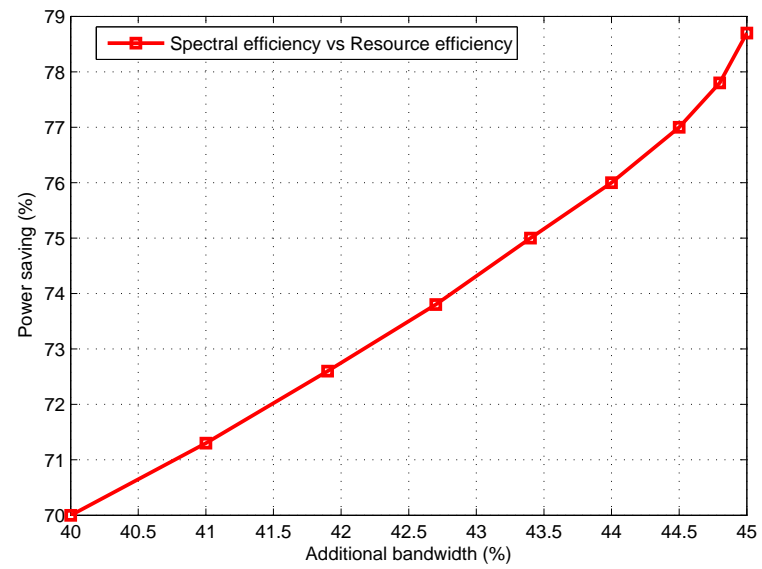

Fig. 11. Spectral efficiency versus resource efficiency for tradeoff between extra bandwidth and power saving. $\left(P_{\max }=1, P_{C}=1, K=4, N=16\right.$ and $W_{C}=1 \mathrm{~Hz}$

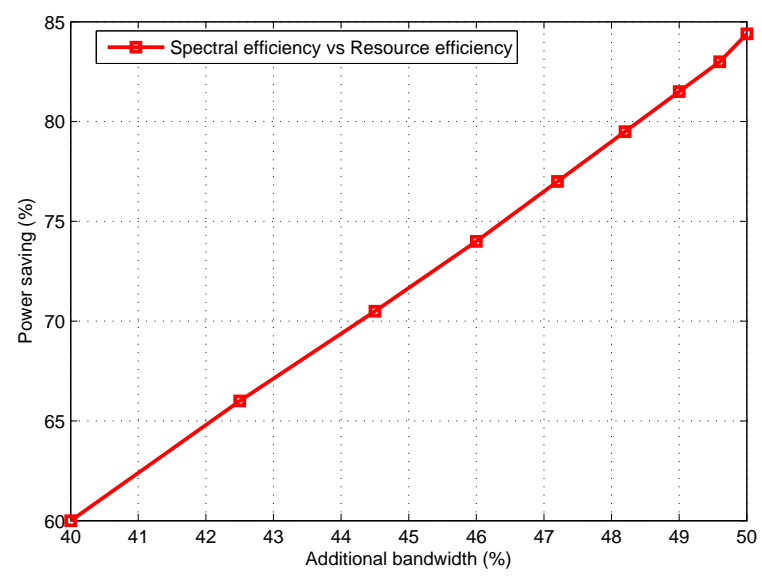

Fig. 12. Spectral efficiency versus resource efficiency for tradeoff between extra bandwidth and power saving. $\left(P_{\max }=1, P_{C}=1, K=50, N=256\right.$ and $W_{C}=1 \mathrm{~Hz}$ )

bandwidth saving. With 16 subcarriers and 4 UEs in the network, Fig. 9 reveals that by using 10\% - 13.5\% extra power compared to optimal energy efficient approach, our proposed scheme is capable of saving $50 \%-66 \%$ bandwidth. With 256 subcarriers and 50 UEs in the network, Fig. 10 reveals that by using $22 \%-27 \%$ extra power compared to optimal energy efficient approach, our proposed scheme is capable of saving $60 \%-80 \%$ bandwidth. Furthermore, Fig. 11 and Fig. 12 show SE versus RE for tradeoff between extra bandwidth and power saving. Fig. 11 reveals that by using $40 \%-45 \%$ extra bandwidth compared to SE based approach, the proposed RE approach is capable of saving 70\%-79\% power. Fig. 12 reveals that with the same parameters as in Fig. 10 by using $40 \%$ - $50 \%$ extra bandwidth, the proposed RE approach is capable of saving $60 \%-85 \%$ power. Therefore, a significant amount of power can be reduced by expanding the bandwidth usage. Thus the proposed RE can optimize the use of available resources in the network. It should also be noted that the amount of power or bandwidth saving can be varied by choosing a different $\beta$. Thus the desirable operation region can be adapted according to the network requirement. 


\section{CONCLUSION}

In this paper, we propose resource efficiency as a new system metric for EE-SE tradeoff in OFDMA based cellular network. We analysis the properties of the proposed RE and provide the optimal and solution. We also provide an upper bound near optimal method to jointly solve the optimization problem. We further develop a suboptimal but low-complexity approach by exploring the inherent structure and property of the resource efficiency approach. Numerical results confirm the theoretical findings and demonstrate the effectiveness of the proposed resource allocation scheme for achieving optimal RE. A major finding is that by using the proposed RE, significant amount of bandwidth can be saved with a slight increase in energy consumption. A similar conclusion can also be drawn on energy saving by bandwidth expansion. This shows that the proposed approach can improve the efficient use of available network resources.

\section{APPENDIX A}

\section{ProOF OF THEOREM I}

Proof: Since subcarrier assignment indicator matrix $\rho$ and its corresponding subcarrier assignment sets $\mathcal{S}_{k}(\forall k \in \mathcal{K})$ are fixed in this case, we can rewrite $\bar{\lambda}_{R E}\left(P_{T}\right)$ as $\frac{\bar{R}\left(P_{T}\right)}{P}+$ $\alpha \bar{R}\left(P_{T}\right)$, where $\alpha=\beta \frac{W_{\text {tot }}}{W P_{\text {tot }}}$. We first prove that $\bar{R}\left(P_{T}\right)$ under the constraints (34)-(36) is strictly concave and continuously differentiable in $P_{T}$. With the nature of water-filling, it is easy to prove that the transmit power on each subcarrier is nondecreasing with the total transmit power. Then we consider the limit under the constraint $\sum_{k \in \mathcal{K}} \sum_{n \in \mathcal{S}_{k}} \triangle p_{k, n}=\triangle P_{T}$. The existence of the limit indicates that $\bar{R}\left(P_{T}\right)$ is continuously differentiable in $P_{T}$ and

$$
\begin{aligned}
\frac{d \bar{R}\left(P_{T}\right)}{d P_{T}} & =\frac{d \bar{R}\left(P_{T}\right)}{d p_{k, n}} \\
& =\max _{k \in \mathcal{K}, n \in \mathcal{S}_{k}} \frac{W_{C} g_{k, n} \log _{2} e}{1+g_{k, n} \bar{p}_{k, n}}
\end{aligned}
$$

Moreover, $\frac{W_{C} g_{k, n} \log _{2} e}{1+g_{k, n} \bar{p}_{k, n}}$ is nonincreasing with $P_{T}$ for $k \in$ $\mathcal{K}$ and $n \in \mathcal{S}_{k}$ while $\max _{k \in \mathcal{K}, n \in \mathcal{S}_{k}} \frac{W_{C} g_{k, n} \log _{2} e}{1+g_{k, n} \bar{p}_{k, n}}$ is strictly monotonically decreasing with $P_{T}$. Thus, $\frac{d^{2} \bar{R}\left(P_{T}\right)}{d P_{T}^{2}}<0$ and $\bar{R}\left(P_{T}\right)$ is strictly concave in $P_{T}$.

In order to prove the quasiconcavity of $\bar{\lambda}_{R E}\left(P_{T}\right)$, we first introduce the definition of quasiconcave function. According to [30], a function $f: \mathbf{R}^{n}-\mathbf{R}$ is called quasiconvex if its domain and all its sublevel sets

$$
\mathcal{S}_{\theta}=\{x \in \operatorname{dom} f \mid f(x) \leq \theta\},
$$

for $\theta \in \mathbf{R}$, are convex. A function is quasiconcave if $-f$ is quasiconvex, i.e., every superlevel set $x \mid f(x) \geq \theta$ is convex. According to [16] and [30], $\frac{\bar{R}\left(P_{T}\right)}{P_{T}}$ is strictly quasiconcave in $P_{T}$ if $\mathcal{S}_{\theta}$ is strictly convex for any real number $\theta$. When $\theta<0$, no points exist on the counter $\frac{\vec{R}\left(P_{T}\right)}{P_{T}}=\theta$. When $\theta \geq 0, \mathcal{S}_{\theta}$ is equivalent to $\mathcal{S}_{\theta}=\left\{P_{T} \geq \sum_{k \in \mathcal{K}} R_{k}^{-1}\left(\mathcal{S}_{k}, \gamma_{k}\right) \mid \theta \zeta P_{T}+\theta P_{C}-\right.$ $\left.\bar{R}\left(P_{T}\right)\right\} \leq 0$, where $R_{k}^{-1}\left(\mathcal{S}_{k}, \gamma_{k}\right)$ is the minimum transmit power required for realizing aggregate rate $\gamma_{k}$ over subcarrier set for the $k^{\text {th }}$ UE. Since $\bar{R}\left(P_{T}\right)$ is strictly concave in $P_{T}$, $\mathcal{S}_{\theta}$ is strictly convex in $P_{T}$. Therefore, $\frac{\bar{R}\left(P_{T}\right)}{P_{T}}$ is continuously differentiable and quasiconcave in $P_{T}$. Since $\bar{R}\left(P_{T}\right)$ is strictly concave in $P_{T}$ and $\frac{\bar{R}\left(P_{T}\right)}{P_{T}}$ is quasiconcave in $P_{T}, \bar{\lambda}_{R E}\left(P_{T}\right)$ is continuously differentiable and quasiconcave in $P_{T}$ and this completes the proof of Property (i).

Furthermore, since $\bar{\lambda}_{R E}\left(P_{T}\right)$ can be rewritten as $\frac{\bar{R}\left(P_{T}\right)}{P}+$ $\alpha \bar{R}\left(P_{T}\right)$, the derivative of RE $\frac{d \bar{\lambda}_{R E}\left(P_{T}\right)}{d P_{T}}$ satisfies $\frac{d \bar{\lambda}_{R E}\left(P_{T}\right)}{d P_{T}}=$ $\frac{d \frac{\bar{R}\left(P_{T}\right)}{P}}{d P_{T}}+\alpha \frac{d \bar{R}\left(P_{T}\right)}{d P_{T}}$. We analysis $\frac{d \frac{\bar{R}\left(P_{T}\right)}{P}}{d P_{T}}$ as follows

$$
\begin{aligned}
\frac{d \frac{\bar{R}\left(P_{T}\right)}{P}}{d P_{T}} & =\lim _{\triangle P_{T} \rightarrow 0} \frac{\frac{\bar{R}\left(P_{T}+\triangle P_{T}\right)}{\zeta\left(P_{T}+\triangle P_{T}\right)+P_{C}}-\frac{\bar{R} P_{T}}{\zeta P_{T}+P_{C}}}{\triangle P_{T}} \\
& =\lim _{\triangle P_{T} \rightarrow 0} \frac{\frac{\bar{R}\left(P_{T}+\triangle P_{T}\right)-\bar{R} P_{T}}{\triangle P_{T}}-\zeta \bar{\lambda}_{E E\left(P_{T}\right)}}{\zeta\left(P_{T}+\triangle P_{T}\right)+P_{C}} \\
& =\frac{\frac{d \bar{R}\left(P_{T}\right)}{d P_{T}}-\zeta \bar{\lambda}_{E E}\left(P_{T}\right)}{\zeta P_{T}+P_{C}}
\end{aligned}
$$

Therefore, we have

$$
\frac{d \bar{\lambda}_{R E}\left(P_{T}\right)}{d P_{T}}=\frac{\left(\beta \frac{\eta_{P}}{\eta_{W}}+1\right) \frac{d \bar{R}\left(P_{T}\right)}{P_{T}}-\zeta \bar{\lambda}_{E E}\left(P_{T}\right)}{\zeta P_{T}+P_{C}}
$$

where $\frac{d \bar{R}\left(P_{T}\right)}{d P_{T}}=\max _{k \in \mathcal{K}, n \in \mathcal{S}_{k}} \frac{W_{C} g_{k, n} \log _{2} e}{1+g_{k, n} \bar{p}_{k, n}}, \bar{\lambda}_{E E}\left(P_{T}\right)=$ $\frac{\bar{R}\left(P_{T}\right)}{\zeta P_{T}+P_{C}}$. This completes the proof of Property (ii) of Theorem I.

\section{APPENDIX B}

\section{ProOF OF THEOREM III}

Proof: For fixed transmission power $P_{T}$, i.e., $P_{T} \in\left[P_{1}, P_{\max }\right]$ where $P_{1}=\sum_{k \in \mathcal{K}} R_{k}^{-1}\left(\mathcal{S}_{k}, \gamma_{k}\right)$ and $R_{k}^{-1}\left(\mathcal{S}_{k}, \gamma_{k}\right)$ is the minimum transmit power required for realizing aggregate rate $\gamma_{k}$ over subcarrier set for the $k^{t h} \mathrm{UE}$ by using uniform power allocation, we have the following proof.

Applying the result form Property $I$, we have $\check{\lambda}_{R E}(W)=$ $\max _{\rho} \frac{\check{R}(W)}{P_{T}}+\bar{\beta} \frac{\check{R}(W)}{W}$, where $\check{R}(W) \triangleq \max _{\rho_{k, n}} R(W)=$ $\max _{\rho_{k, n}} \sum_{k \in \mathcal{K}} \sum_{n \in \mathcal{N}} \rho_{k, n} r_{k, n}$, under the constraint of (58)(60), and hence

$$
\frac{d \check{\lambda}_{R E}(W)}{d W}=\frac{1}{P_{T}} \frac{d \check{R}(W)}{d W}+\frac{\bar{\beta}}{W} \frac{d \check{R}(W)}{d W}-\frac{\bar{\beta} \check{R}(W)}{W^{2}}
$$

Therefore, we have

$$
\begin{aligned}
\frac{d^{2} \check{\lambda}_{R E}(W)}{d W^{2}} & =\frac{1}{P_{T}} \frac{d^{2} \check{R}(W)}{d W^{2}}+\frac{\bar{\beta}}{W} \frac{d^{2} \check{R}(W)}{d W^{2}}-\frac{\bar{\beta}}{W^{2}} \frac{d \check{R}(W)}{d W} \\
& +\frac{2 \bar{\beta} \check{R}(W)}{W^{3}}-\frac{\bar{\beta}}{W^{2}} \frac{d \check{R}(W)}{d W} \\
& =\left(\frac{1}{P_{T}}+\frac{\bar{\beta}}{W}\right) \frac{d^{2} \check{R}(W)}{d W^{2}} \\
& +\frac{2 \bar{\beta}}{W^{2}}\left(\frac{\check{R}(W)}{W}-\frac{d \check{R}(W)}{d W}\right)
\end{aligned}
$$

Since $W=Q \times W_{C}=\sum_{k \in \mathcal{K}} \sum_{n \in \mathcal{N}} \rho_{k, n} W_{C}$, we have

$$
d W=W_{C} d \rho_{k, n} \text { and } d \rho_{k, n}=\frac{d W}{W_{C}} .
$$


Furthermore, $\rho_{k, n} \in\{1,0\}$ indicates whether or not the $n^{t h}$ subcarrier is assigned to the $k^{t h}$ UE. Hence $W$ is not continuous and we need to relax $\rho_{k, n}$ as follows

$$
0 \leq \rho_{k, n} \leq 1 \text { and } Q=\sum_{k \in \mathcal{K}} \sum_{n \in \mathcal{N}} \rho_{k, n} .
$$

Thus, we now analyze the term $\frac{d \check{R}(W)}{d W}$ as follows

$$
\begin{aligned}
\frac{d \check{R}(W)}{d W} & =\frac{d \max _{\rho} \sum_{k \in \mathcal{K}} \sum_{n \in \mathcal{N}} \rho_{k, n} W_{C} \log _{2}\left(1+p_{k, n} g_{k, n}\right)}{W_{C} d \rho_{k, n}} \\
& =\max _{\rho_{k, n}} \log _{2}\left(1+p_{k, n} g_{k, n}\right)+\max _{\rho_{k, n}} \frac{\rho_{k, n} g_{k, n} \frac{d p_{k, n}}{d \rho_{k, n}} \log _{2} e}{1+p_{k, n} g_{k, n}} .
\end{aligned}
$$

With uniform power allocation scheme and subcarrier maximum assignment scheme, $p_{k, n}=\rho_{k, n} \frac{P_{T}}{Q}=\rho_{k, n} \frac{P_{T} W_{C}}{W}$, (73) can be rewritten as follows

$$
\begin{aligned}
\frac{d \check{R}(W)}{d W} & =\log _{2}\left(1+\frac{P_{T} W_{C}}{W} \check{g}_{k, n}\right)+\frac{P_{T}\left(W-W_{C}\right) \log _{2} e}{P_{T} W+\frac{W^{2}}{\check{g}_{k, n} W_{C}}} \\
& =\log _{2}\left(1+\frac{P_{T}}{W} f_{k, n}\right)+\frac{P_{T}\left(W-W_{C}\right) \log _{2} e}{P_{T} W+\frac{W^{2}}{f_{k, n}}}
\end{aligned}
$$

where $\check{g}_{k, n}=\max _{k \in \mathcal{K}, n \in \mathcal{N}^{\prime}} g_{k, n}$ and $f_{k, n}=\check{g}_{k, n} W_{C}$. Based on this result, we now have $\frac{d^{2} \check{R}(W)}{d W^{2}}$ as follows

$$
\frac{d \check{R}^{2}(W)}{d W^{2}}=\left(-\frac{1}{W}+\frac{W_{C}}{W^{2}}+\frac{W_{C}-W}{W^{2}+P_{T} W f_{k, n}}\right) \frac{\log _{2} e}{1+\frac{W}{P_{T} f_{k, n}}}
$$

We then analysis $\frac{\check{R}(W)}{W}-\frac{d \check{R}(W)}{d W}$ as (76) in the next page, Since $\Upsilon \leq 0$, we have

$$
\frac{\check{R}(W)}{W}-\frac{d \check{R}(W)}{d W} \leq \frac{P_{T}\left(W_{C}-W\right) \log _{2} e}{P_{T} W+\frac{W^{2}}{f_{k, n}}}
$$

Substituting (75) and (77) in (70), we have (78) which is shown in the next page. Since $\frac{3 \bar{\beta} \log _{2} e}{W^{2}\left(1+\frac{W}{P_{T} f_{k, n}}\right)}$ is always positive, the $\operatorname{sign}\left(\frac{d^{2} \check{\lambda}_{R E}(W)}{d W^{2}}\right)$ depends on

$$
\operatorname{sign}\left(\frac{d^{2} \check{\lambda}_{R E}(W)}{d W^{2}}\right)=\frac{W_{C}}{W}-1=\frac{1}{Q}-1 .
$$

Since $Q \geq 1, \operatorname{sign}\left(\frac{d^{2} \check{\lambda}_{R E}(W)}{d W^{2}}\right)<0$. Therefore, $\check{\lambda}_{R E}(W)$ either strictly decreases or first strictly increases and then strictly decreases with $Q$, this completes the proof of Theorem III.

\section{APPENDix C}

\section{PROOF OF THEOREM IV}

Proof: Since subcarrier assignment indicator matrix $\rho$ and its corresponding subcarrier assignment sets $\mathcal{S}_{k}(\forall k \in \mathcal{K})$ are fixed in this case, we rewrite $\hat{\lambda}_{R E}\left(P_{T}\right)$ as $\frac{\hat{R}\left(P_{T}\right)}{P}+\alpha \hat{R}\left(P_{T}\right)$, where $\hat{R}\left(P_{T}\right) \triangleq \max _{>0} \sum_{k \in \mathcal{K}} \sum_{n \in \mathcal{S}_{k}} r_{k, n}$, under the constraint of (62)-(63). "We first prove that $\hat{R}\left(P_{T}\right)$ is strictly concave and continuously differentiable in $P_{T}$. We consider the limit under the constraint $\sum_{k \in \mathcal{K}} \sum_{n \in \mathcal{S}_{k}} \triangle p_{k, n}=\triangle P_{T}$, the existence of the limit indicates that $\hat{R}\left(P_{T}\right)$ is continuously differentiable in $P_{T}$. Then we analyze $\frac{d \hat{R}\left(P_{T}\right)}{d P_{T}}$ as follows

$$
\frac{d \hat{R}\left(P_{T}\right)}{d P_{T}}=\frac{d \max _{p_{k, n} \geq 0} \sum_{k \in \mathcal{K}} \sum_{n \in \mathcal{S}_{k}} W_{C} \log _{2}\left(1+p_{k, n} g_{k, n}\right)}{d P_{T}} .
$$

Substitute (63) into (80), we have

$$
\begin{aligned}
\frac{d \hat{R}\left(P_{T}\right)}{d P_{T}} & =\frac{d \sum_{k \in \mathcal{K}} \sum_{n \in \mathcal{S}_{k}} W_{C} \log _{2}\left(1+\frac{P_{T}}{Q} g_{k, n}\right)}{d P_{T}} \\
& =\sum_{k \in \mathcal{K}} \sum_{n \in \mathcal{S}_{k}} \frac{W_{C} \log _{2} e}{\frac{Q}{g_{k, n}}+P_{T}} .
\end{aligned}
$$

Since $\frac{W_{C} \log _{2} e}{\frac{Q}{g_{k, n}}+P_{T}}$ is decreasing with $P_{T}$ for $k \in \mathcal{K}$ and $n \in \mathcal{S}_{k}, \sum_{k \in \mathcal{K}} \sum_{n \in \mathcal{S}_{k}} \frac{W_{C} \log _{2} e}{\frac{Q}{g_{k, n}}+P_{T}}$ is strictly monotonically decreasing with $P_{T}$. Thus, $\frac{d^{2} \hat{R}\left(P_{T}\right)}{d P_{T}^{2}}<0$ and $\hat{R}\left(P_{T}\right)$ is strictly concave in $P_{T}$.

Similar as the proof of Theorem $I, \frac{\hat{R}\left(P_{T}\right)}{P_{T}}$ is strictly quasiconcave in $P_{T}$ if $\mathcal{S}_{\sigma}$ is strictly convex for any real number $\sigma$. When $\sigma<0$, no points exist on the counter $\frac{\hat{R}\left(P_{T}\right)}{P_{T}}=\sigma$. When $\sigma \geq 0, \mathcal{S}_{\sigma}$ is equivalent to $\mathcal{S}_{\sigma}=$ $\left\{P_{T} \geq \sum_{k \in \mathcal{K}} R_{k}^{-1}\left(\mathcal{S}_{k}, \gamma_{k}\right) \mid \sigma \zeta P_{T}+\sigma P_{C}-\hat{R}\left(P_{T}\right)\right\} \leq 0$, where $R_{k}^{-1}\left(\mathcal{S}_{k}, \gamma_{k}\right)$ is the minimum transmit power required for realizing aggregate rate $\gamma_{k}$ over subcarrier set for the $k^{\text {th }}$ UE by using uniform power allocation. Since $\hat{R}\left(P_{T}\right)$ is strictly concave in $P_{T}, \frac{\hat{R}\left(P_{T}\right)}{P_{T}}$ is continuously differentiable and quasiconcave in $P_{T}$. Therefore, $\hat{\lambda}_{R E}\left(P_{T}\right)$ is continuously differentiable and quasiconcave in $P_{T}$.

Hence, the derivative of $\operatorname{RE} \frac{d \hat{\lambda}_{R E}\left(P_{T}\right)}{d P_{T}}$ satisfies

$$
\frac{d \hat{\lambda}_{R E}\left(P_{T}\right)}{d P_{T}}=\frac{d \frac{\hat{R}\left(P_{T}\right)}{P}}{d P_{T}}+\beta \frac{W_{t o t}}{W P_{t o t}} \frac{d \hat{R}\left(P_{T}\right)}{d P_{T}}
$$

Substituting (67) and (81) into (82), we have

$\frac{d \hat{\lambda}_{R E}\left(P_{T}\right)}{d P_{T}}=\frac{\left(\beta \frac{\eta_{P}}{\eta_{W}}+1\right) \sum_{k \in \mathcal{K}} \sum_{n \in \mathcal{S}_{k}} \frac{W_{C} \log _{2} e}{\frac{Q}{g_{k, n}}+P_{T}}-\zeta \hat{\lambda}_{E E}\left(P_{T}\right)}{\zeta P_{T}+P_{C}}$

where $\hat{\lambda}_{E E}\left(P_{T}\right)=\frac{\sum_{k \in \mathcal{K}} \sum_{n \in \mathcal{S}_{k}} W_{C} \log _{2}\left(1+\frac{P_{T}}{Q} g_{k, n}\right)}{\zeta P_{T}+P_{C}}$ and this completes the proof of Theorem $I V$.

\section{REFERENCES}

[1] M. Kang, M. S. Alouini, and L. Yang, "Outage probability and spectrum efficiency of cellular mobile radio systems with smart antennas," IEEE Trans. Commun., vol. 50, no. 7, pp. 1871 - 1877, Dec. 2002.

[2] H. Dai and H. Poor, "Asymptotic spectral efficiency of multi-cell MIMO systems with frequency-flat fading," IEEE Trans. Signal Process., vol. 51, pp. 2976 - 2988, Nov. 2003.

[3] S. Verdu, "Spectral efficiency in the wideband regime," IEEE Trans. Inf. Theory, vol. 48, no. 6, pp. 1319 - 1343, June 2002.

[4] R. S. Prabhu and B. Daneshrad, "Performance analysis of energyefficient power allocation for MIMO-MRC systems," IEEE Trans. Commun., vol. 60, no. 8, pp. 2048 - 2053, Aug. 2012.

[5] J. Xu and L. Qiu, "Energy efficiency optimization for MIMO broadcast channels," IEEE Trans. Wireless Commun., vol. 12, no. 2, pp. $690-$ 701, Feb. 2013.

[6] G. Koutitas, A. Karousos, and L. Tassiulas, "Deployment strategies and energy efficiency of cellular networks," IEEE Trans. Wireless Commun., vol. 11, no. 7, pp. 2552 - 2563, July 2012.

[7] C. Ghosh and S. Roy, "Coexistence challenges for heterogeneous cognitive wireless networks in TV white spaces," IEEE Wireless Communications, vol. 18, no. 4, pp. 22 - 31, August 2011. 


$$
\frac{\check{R}(W)}{W}-\frac{d \check{R}(W)}{d W}=\underbrace{\frac{\max _{\boldsymbol{\rho}} \sum_{k \in \mathcal{K}} \sum_{n \in \mathcal{N}} \rho_{k, n} W_{C} \log _{2}\left(1+p_{k, n} g_{k, n}\right)}{\sum_{k \in \mathcal{K}} \sum_{n \in \mathcal{N}} \rho_{k, n} W_{C}}-\max _{\rho_{k, n}} \log _{2}\left(1+p_{k, n} g_{k, n}\right)}_{\Upsilon}+\frac{P_{T}\left(W_{C}-W\right) \log _{2} e}{P_{T} W}+\frac{W^{2}}{f_{k, n}}
$$

$$
\begin{aligned}
\frac{d^{2} \check{\lambda}_{R E}(W)}{d W^{2}} & =\left(\frac{1}{P_{T}}+\frac{\bar{\beta}}{W}\right) \frac{d^{2} \check{R}(W)}{d W^{2}}+\frac{2 \bar{\beta}}{W^{2}}\left(\frac{\check{R}(W)}{W}-\frac{d \check{R}(W)}{d W}\right) \\
& <\frac{\bar{\beta}}{W}\left(-\frac{1}{W}+\frac{W_{C}}{W^{2}}+\frac{W_{C}-W}{W^{2}+P_{T} W f_{k, n}}\right) \frac{\log _{2} e}{1+\frac{W}{P_{T} f_{k, n}}}+\frac{2 \bar{\beta}}{W^{2}} \frac{P_{T}\left(W_{C}-W\right) \log _{2} e}{P_{T} W+\frac{W^{2}}{f_{k, n}}} \\
& =\frac{\bar{\beta}}{W}\left(-\frac{1}{W}+\frac{W_{C}}{W^{2}}+\frac{W_{C}-W}{W^{2}+P_{T} W f_{k, n}}\right) \frac{\log _{2} e}{1+\frac{W}{P_{T} f_{k, n}}}+\frac{2 \bar{\beta}\left(W_{C}-W\right)}{W^{3}} \frac{\log _{2} e}{1+\frac{W}{P_{T} f_{k, n}}} \\
& =\frac{\log _{2} e}{1+\frac{W}{P_{T} f_{k, n}}}\left[\frac{2 \bar{\beta}\left(W_{C}-W\right)}{W^{3}}+\frac{\bar{\beta}}{W}\left(-\frac{1}{W}+\frac{W_{C}}{W^{2}}+\frac{W_{C}-W}{W^{2}+P_{T} W f_{k, n}}\right)\right] \\
& \leq \frac{\log _{2} e}{1+\frac{W}{P_{T} f_{k, n}}}\left[\frac{2 \bar{\beta}\left(W_{C}-W\right)}{W^{3}}+\frac{\bar{\beta}}{W}\left(\frac{W_{C}}{W^{2}}-\frac{1}{W}\right)\right] \\
& =\frac{3 \bar{\beta} \log _{2} e}{W^{2}\left(1+\frac{W}{P_{T} f_{k, n}}\right)}\left(\frac{W_{C}}{W}-1\right) .
\end{aligned}
$$

[8] Z. Hasan, H. Boostanimehr, and V. K. Bhargava, "Green cellular networks: A survey, some research issues and challenges," IEEE Commun. Surveys Tutorials, vol. 13, no. 4, pp. 524 - 540, quarter 2011.

[9] G. W. Miao, N. Himayat, G. Y. Li, and D. Bormann, "Energy efficient design in wireless OFDMA," in Proc. IEEE Int. Conf. Commun., pp. 3307 - 3312, May 2008.

[10] D. W. K. Ng, E. S. Lo, and R. Schober, "Energy-efficient resource allocation in multi-cell OFDMA systems with limited backhaul capacity," IEEE Trans. Wireless Commun., vol. 11, no. 10, pp. 3618 - 3631, Oct. 2012.

[11] D. W. K. Ng, E. S. Lo, and R. Schober, "Energy-efficient resource allocation in OFDMA systems with large numbers of base station antennas," IEEE Trans. Wireless Commun., vol. 11, no. 9, pp. 3292 - 3304, Sep. 2012.

[12] X. Xiao, X. Tao, and J. Lu, "Qos-aware energy-efficient radio resource scheduling in multi-user OFDMA systems," IEEE Commun. Lett., vol. 17 , no. 1 , pp. $75-78$, Jan. 2013.

[13] G. Miao, N. Himayat, G. Y. Li, and S. Talwar, "Low-complexity energyefficient scheduling for uplink OFDMA," IEEE Trans. Commun., vol. 60, no. 1, pp. 112 - 120, Jan. 2012.

[14] S. Buzzi, G. Colavolpe, D. Saturnino, and A. Zappone, "Potential games for energy-efficient power control and subcarrier allocation in uplink multicell OFDMA systems," IEEE Journal OF Selected Topics in Signal Processing, vol. 6, no. 2, pp. 89 - 103, APRIL 2012.

[15] C. Xiong, G. Li, Y. Liu, Y. Chen, and S. Xu, "Qos-aware energy-efficient radio resource scheduling in multi-user OFDMA systems," IEEE Trans. Wireless Commun., vol. 12, no. 6, pp. 3085 - 3095, June 2013.

[16] C. Xiong, G. Li, S. Zhang, Y. Chen, and S. Xu, "Energy-efficient resource allocation in OFDMA networks," IEEE Trans. Commun., vol. 60 , no. 12 , pp. 3767 - 3778, Dec. 2012.

[17] F. Meshkati, H. V. Poor, and S. C. Schwartz, "Energy-efficient resource allocation in wireless networks," IEEE Signal Process. Mag., vol. 24 no. 3, pp. 58 - 68, May 2007.

[18] G. Miao, N. Himayat, G. Y. Li, and A. Swami, "Cross-layer optimization for energy-efficient wireless communications: a survey," Wiley J. Wireless Commun. Mobile Comput., vol. 9, no. 4, pp. 529 - 542, Apr. 2009.

[19] Y. Chen, S. Zhang, S. Xu, and G. Y. Li, "Fundamental tradeoffs on green wireless networks," IEEE Commun. Mag., vol. 49, no. 6, pp. 30 - 37, June 2011.

[20] Y. Li, M. Sheng, C. Yang, and X. Wang, "Energy efficiency and spectral efficiency tradeoff in interference-limited wireless networks," IEEE Commun. Lett., vol. 17, no. 10, pp. 1924 - 1927, Oct. 2013.
[21] C. Bae and W. E. Stark, "End-to-end energy-bandwidth tradeoff in multihop wireless networks," IEEE Trans. Inf. Theory, vol. 55, no. 9, pp. 4051 - 4066, Sep. 2009.

[22] O. Onireti, F. Heliot, and M. A. Imran, "On the energy efficiency-spectral efficiency trade-off in the uplink of CoMP system," IEEE Trans. Wireless Commun., vol. 11, no. 2, pp. 556 - 561, Feb. 2012.

[23] C. Xiong, G. Li, S. Zhang, Y. Chen, and S. Xu, "Energy- and spectralefficiency tradeoff in downlink OFDMA networks," IEEE Trans. Wireless Commun., vol. 10, no. 11, pp. 3874 - 3886, Nov. 2011.

[24] S. Cui, A. Goldsmith, and A. Bahai, "Energy-constrained modulation optimization," IEEE Trans. Wireless Commun., vol. 4, no. 5, pp. 2349 -2360 , Sep. 2005.

[25] G. B. L. C. A. C. Coello and D. A. V. Veldhuizen, Evolutionary Algorithms for Solving Multi-Objective Problems. New York: Springer, 2007.

[26] C. Y. Wong, R. S. Cheng, K. B. Lataief, and R. D. Murch, "Multiuser OFDM with adaptive subcarrier, bit, and power allocation," IEEE Journal on Selected Areas in Commun., vol. 17, no. 10, pp. 1747 1758, Oct. 1999.

[27] W. Yu and R. Lui, "Dual methods for nonconvex spectrum optimization of multicarrier systems," IEEE Trans on Commun., vol. 54, no. 7, pp. $1310-1322$, July 2006.

[28] W. Yu and J. M. Coffi, "FMDA capacity of Gaussian multiple-access channel with ISI," IEEE Trans on Commun., vol. 50, no. 1, pp. $102-$ $111,2002$.

[29] S. Boyd, Branch and Bound Methods. Stanford University.

[30] S. Boyd and L. Vandenberghe, Convex Optimization. Cambridge University Press, Cambridge, UK, 2004. 